\title{
GENERATING OPTIMAL TOPOLOGIES IN STRUCTURAL DESIGN USING A HOMOGENIZATION METHOD
}

\author{
Martin Philip BENDSøE \\ Mathematical Institute, The Technical University of Denmark, DK-2800 Lyngby, Denmark
}

and

Noboru KIKUCHI

Applied Mechanics Department, Computational Mechanics Laboratory, University of Michigan, Ann Arbor, MI 48109, U.S.A.

Received 28 March 1988

\begin{abstract}
Optimal shape design of structural elements based on boundary variations results in final designs that are topologically equivalent to the initial choice of design, and general, stable computational schemes for this approach often require some kind of remeshing of the finite element approximation of the analysis problem. This paper presents a methodology for optimal shape design where both these drawbacks can be avoided. The method is related to modern production techniques and consists of computing the optimal distribution in space of an anisotropic material that is constructed by introducing an infimum of periodically distributed small holes in a given homogeneous, isotropic material, with the requirement that the resulting structure can carry the given loads as well as satisfy other design requirements. The computation of effective material properties for the anisotropic material is carried out using the method of homogenization. Computational results are presented and compared with results obtained by boundary variations.
\end{abstract}

\section{Introduction}

Shape optimization of linearly elastic structures has been studied for more than fifteen years and has reached a level of maturity that makes it viable to implement the methods in CAE (computer aided engineering) systems for production use. For example, several software vendors of finite element general-purpose programs are introducing modules of shape optimization based on the successful implementation of sensitivity analysis for sizing design optimization problems in such codes. However, shape optimization is quite different from sizing problems. For sizing optimization problems, once sensitivity is known, design modification is rather simple, and a corresponding modification of a finite element model is straightforward. Computing sensitivity is the same for shape sensitivity as for sizing problems if the shape of a structure is defined by a parametric equation using e.g. spline functions. In this sense, shape optimization is not different from sizing problems, but the shape change also implies significant change of the corresponding finite element model so that sensitivity analysis itself is not sufficient for shape optimization of structures. Therefore, unless finite element analysis 
and its preprocessing for mesh generation are fully integrated in a shape optimization module, it is quite difficult to utilize sensitivity analysis in practical shape optimization problems. Since most finite element analysis programs are intended to be independent of preprocessing, a shape optimization module in these programs cannot be easily implemented, whereas sensitivity analysis is more or less straightforward. In other words, shape optimization algorithms may be installed in either a mesh generation or a geometric modeling module under the assumption that sensitivity with respect to design is calculated in the finite element analysis module. Even in this setting, there still remains a serious difficulty in that the topology of the optimal shape is unknown a priori. This means that choice of some basis functions to describe the boundary of a structure at the beginning of the optimal design process determines a restricted class of the optimal solutions that can be achieved. Aiso, it is very difficult to change the topology of a structure during the design process. If a singly connected domain is assumed at the beginning, the optimal shape that is obtained is within this class of domains, although the true optimal shape may be doubly connected, or perhaps even a structure with microvoids. This can only be avoided if an algorithm that automatically changes the topology of the finite element models is invoked.

The development of the boundary movement methodology for shape optimization has attracted a great deal of attention and the literature on the subject is quite extensive; we refer to the survey [1] by Haftka and Gandhi. The boundary variation method can be implemented in various ways, for example by employing certain mesh moving schemes to define the shape of a given structure (see for example [2]). In this case the design variables are the coordinates of nodal points of a finite element model of a structure. Such schemes usually require some method that will maintain "regularity" of finite elements near the boundary, as in [3] where nodal points on the design boundary are moved in the normal direction of the current design. Also, Kikuchi et al. showed in [4] that "regularity" of the finite element model is very important near the design boundary so as to yield a physically sound optimal shape using these schemes. A different approach to represent boundaries in shape optimization is to introduce the boundary segment idea which describes the design boundary by a set of simple segments such as straight lines, circular arcs, elliptic arcs, and splines. The optimum is then sought within this restricted definition of the boundary. The idea is particularly suitable to geometric modeling in CAE systems, and is extensively applied in solving practical shape optimization problems. The method was used by Tvergaard [5] in 1973 and has had a revival in recent years, c.f. [6,7]. For this method "regularity" of finite element mesh is also of great importance, especially in cases where large design changes occur. This can be handled by adaptive schemes as in [4] or by automatic remeshing of the finite elemlent model at each design iteration (see $[6,8]$ ).

The mathematical foundation for optimal shape design and design sensitivity analysis in boundary variation methods is well established. Sensitivity analysis for shape optimization problems is thoroughly studied in various settings by, for example, Haug, Choi and Komkov [9], Choi and Haug [10], Choi and Seong [11], Rousselet and Haug [12], Zolesio [13], Simon [14], and Haber [15], and these results have been applied to solve various shape optimization problems. The problem of existence of solutions has been addressed by Chenais [16].

The state-of-the-art today for shape design is that shape optimization is possible under the assumption that the initial topology is fixed during the iterative design optimization. What is the next step in shape optimization? An answer to this could be the introduction of a new 
method that can yield the optimal topology as well as the optimal shape of a structure. To do this, it is too difficult to use the methods that describe the design boundary using parametric equations, since change of topology cannot be expected in the design process. In other words it is necessary to represent the shape without using "shape" functions. The method introduced in this paper is a possible alternative approach to shape optimization. Roughly speaking, shape optimization problems are transformed to material distribution problems using composite materials. Two material constituants, substance and void, are considered, and the microscopic optimal void distribution is considered instead of shape optimization by boundary variations in the usual sense. An important feature of the procedure is that the homogenization method is applied to determine macroscopic constitutive equations for the material with microscopic material constituants.

Materials with microstructure enter naturally in problems of optimal structural design, be it shape or sizing problems. This was first clearly demonstrated in the papers by Cheng and Olhoff $[17,18]$ on optimal thickness distribution for elastic plates, although some indications of this phenomena can be found in earlier papers by Cea et al. [19] and Tartar [20]. The work by Olhoff and Cheng led to a series of works on optimal design problems introducing microstrucutres in the formulation of the problem. Most work has related to the plate problem [21-24] and to the problem of optimal design of torsion bars constructed from two dissimilar materials in a given volume fraction $[23,25,26]$. The design of a beam using a twodimensional model is described in Zochowski [27]. The plate design problem is a sizing problem while the torsion bar problem is a shape design problem (designing the shape of inclusion of a weak material in a strong material). In both cases it turns out that laminated structures give more efficient designs and thus microstructures have to be built up in order to obtain the strongest structures. This requires a consistent way for computing effective material properties for materials with microstructures and this can conveniently be carried out using the method of homogenization [20,28-31]. Thus optimal design of structures is closely connected with studies of materials with microstructures and the very important problem of finding (optimal) bounds on the effective material properties for composites [32-38]. In mathematical terms the introduction of microstructures in the formulation of structural design problems corresponds to a relaxation of the variational problem that can be formulated for the design optimization, see e.g. $[39,40]$.

The design method described in this paper is strongly inspired by the works mentioned above as well as being related to modern production techniques such as numerically controlled milling and plastic forming with controlled porosity through controlled cooling. We take an approach where a structural element is understood in a broad sense a being defined only by the loads it is supposed to carry, its volume (cost), and design require, ents as stress and strain limitations. The only restrictions on the allowable shapes is that the el $t$ ment should connect to the given surface tractions. The initial design in the iterative design optimization procedure is a rough block of space in which we fill material in an optimal way (or we have a rough block of material and remove material). The use of a fixed domain of simple geometry simplifies the construction of a finite element approximation and the necessity of remeshing is avoided.

The nature of the method is such that it allows you to predict the topology of the siructural member but it only results in a nonsmooth estimate of the exact form of its boundary. The method should be the first step in a two-step shape design procedure, where the second step consists of a traditional boundary variations optimization, based on the design computed in 
the first step. This step will be speeded up considerably, as the first step gives a boundary close to the optimal, smooth boundary. Also, the first step will result in estimates of stresses and strains in the structure that will allow construction of an effective finite element mesh for the boundary optimization.

\section{General problem formulation for optimal design of linearly elastic structures}

In the following a general formulation for optimal structural design in linear elasticity is presented. The set-up is well known, but will be repeated here in order to introduce the notation that will be used as well as to clarify ideas.

Consider a mechanical element as a body occupying a domain $\Omega$ in $\mathbb{R}^{3}$ and suppose that the body is subject to body forces $f$ and boundary tractions $t$. In optimal design for minimum compliance we seek the optimal choice of elasticity tensor $E_{i j k l}$ in some given set of admissable elasticity tensors, $U_{\text {ad }}$. The admissible tensors will usually be allowed to vary over the domain of the body, so that $E_{i j k l}$ will be a function of the spatial variable $x \in \Omega$, and we have $U_{\mathrm{ad}} \in\left(L^{\infty}(\Omega)\right)^{21}$, in general, corresponding to the 21 independent elements of $E_{i j k l}$.

Introducing the energy bilinear form

$$
a_{E}(u, v)=\int_{\Omega} E_{i j k l} \varepsilon_{k l}(u) \varepsilon_{i j}(v) \mathrm{d} x,
$$

with linearized strains

$$
\varepsilon_{i j}(u)=\frac{1}{2}\left(\frac{\partial u_{i}}{\partial x_{j}}+\frac{\partial u_{j}}{\partial x_{i}}\right),
$$

and the load linear form

$$
L(v)=\int_{\Omega} f \cdot v \mathrm{~d} x+\int_{\Gamma_{\mathrm{T}}} t \cdot v \mathrm{~d} s,
$$

the minimum compliance problem takes the form:

$$
\begin{aligned}
& \operatorname{minimize} L(u) \\
& E_{i j k l} \in U_{a d}
\end{aligned}
$$

subject to $a_{\mathrm{E}}(u, v)=L(v)$ all $v \in U$, design constraints .

Here "design constraints" covers constraints on stresses, strains, displacements, etc., while sizing constraints, volume constraints, etc. are counted for in the choice of $U_{\mathrm{ad}}$. The space $U$ is the space of kirematically admissible displacement fields.

In the case of optimal shape design elements $E_{i j k l}$ of $U_{\text {ad }}$ take on the form

$$
E_{i j k l}(x)=\chi(x) \bar{E}_{i j k l},
$$


where $\bar{E}_{i j k l}$ is the constant elastic tensor for the material employed for the construction of the mechanical element, and $\chi(x)$ is an indicator function for the part $\Omega^{\mathrm{m}}$ of $\Omega$ that is occupied by the material:

$$
\chi(x)=\left\{\begin{array}{ll}
1 & \text { if } x \in \Omega^{m}, \\
0 & \text { if } x \in \Omega^{m}, \Omega^{m}
\end{array} .\right.
$$

For sizing problems, like design of variable thickness sheets, the admissible $E_{i j k l}$ 's have the form

$$
E_{i j k l}(x)=h(x) \bar{E}_{i j k l},
$$

where again $\bar{E}_{i j k l}$ is a constant tensor and $h \in L^{\infty}(\Omega)$ is the sizing function.

In the two examples above, it is natural to impose a volume constraint, and this would take on the form

$$
\int_{\Omega} \chi(x) \mathrm{d} x=\text { Vol }, \quad \int_{\Omega} h(x) \mathrm{d} x=\text { Vol } .
$$

Of the two design problems described above, the sizing problem is well posed, solutions exist [41], and for computations this problem is straightforward. However, the shape design problem as posed does not, in general, have a solution (cf. [39]) unless you regularize the problem in some way by introducing composite materials. Traditionally shape design problems are treated in a different way by defining shapes as given by mappings into $\mathbb{R}^{3}$, defined on a given reference domain $\Omega_{0}$ in $\mathbb{R}^{3}$. Because of smoothness properties required in this method, the class of shapes that is considered will be diffeomorfic with the reference domain $\boldsymbol{\Omega}_{0}$ and shape changes will be boundary movements. In the mapping method the mechanical element is thus defined as $\varphi\left(\Omega_{0}\right)$, where $\varphi$ is a diffeomorfism, $\varphi: \Omega_{0} \rightarrow \varphi\left(\Omega_{0}\right)$ and $\Omega_{0}$ in $\mathbb{R}^{3}$. By giving deformation fields etc. on the body $\varphi\left(\Omega_{0}\right)$ in terms of deformation fields on the reference domain $\Omega_{0}$, shape design by boundary variations can also be stated as in (4) on the reference domain $\Omega_{0}$ (see for example $[12,13,14]$ ). In this way the energy bilinear form as well as the load linear form will depend on the derivatives of the design variable $\varphi \in C^{1}\left(\Omega_{0}\right.$, $\left.\mathbb{R}^{3}\right)$. The linear form $L$ will have the form

$$
L(v)=\int_{\Omega_{0}} f \cdot v\left|\operatorname{det} D \varphi^{-1}\right| \mathrm{d} x+\int_{\Gamma_{\mathrm{T} 0}} t \cdot v\left|\operatorname{adj} D \varphi^{-1}\right| \mathrm{d} s,
$$

where $D \varphi$ denotes the Jacobian of the mapping $\varphi$. The energy bilinear form will have the form as given in (1), with the elasticity tensor $E_{i j k l}$,

$$
E_{i j k l}=E_{i p k q}\left(D \varphi^{-1}\right)_{j p}\left(D \varphi^{-1}\right)_{l q}|\operatorname{det} D \varphi| .
$$

It can readily be seen from this that a moving boundary technique leads to a complicated functional dependence on the derivatives of the design function $\varphi$, and this property has to be taken into account in any discretized, numerical method for solving problems of this type. 
As discussed in the introduction this method actually has serious computational drawbacks, as well as being unable, in a rational way, to predict the eventual optimal topology of the mechanical element. In order to circumvent this unfortunate situation we thus need an approach which in principle is related to the general shape design statement used above and which has the computational attractive simplicity of the sizing problem. The method that we propose is to use composites of a priori simple form which allows you to describe the body by a density function that can take on values in the interval $[0,1]$ instead of only the values 0 and 1 as for the indicator function statement above. This results in a problem that in nature is a sizing problem, and which at the same time allows you to predict the optimal distribution of material, that is, predict the optimal shape.

The method consists of the following steps:

Step 1. Choose a suitable reference domain that allows you to define surface tractions, fixed boundaries, etc.

Step 2. Choose a composite, constructed by period repetition of a unit cell consisting of the given material with one or more holes.

Step 3. Compute the effective material properties of the composite, using homogenization theory. This gives a functional relationship between the density of material in the composite (i.e. sizes of holes) and the effective material properties.

Step 4. Compute the optimal distribution of this composite material in the reference domain, treating the problem as a sizing problem with the density as the sizing variable

Step 5. Interpret the optimal distribution of material as defining a shape, in the sense of the general shape design formulation given above.

\section{The homogenization method}

In this section the homogenization method will be briefly reviewed for the sake of completeness of the paper. Details of homogenization methods can be found in the references sited in the introduction [28-31]; for homogenization of elasticity problems see also [42, 43]. Numerical studies of homogenization can be found in $[44,45]$. For simplicity, plane problems are considered here.

Suppose that a periodic microstructure is assumed in a neighborhood of an arbitrary point $x$ of a given linearly elastic structure. The periodicity is represented by a parameter $\varepsilon$ which is assumed to be very small, that is, the elasticity tensor is given by $E_{i j k l}^{\varepsilon}(x)$, where

$$
E_{i j k l}^{\varepsilon}(x)=E_{i j k l}(x, y), \quad y=x / \varepsilon, \text { for } i, j, k, l=1,2,
$$

and $y \rightarrow E_{i j k l}(x, y)$ is $Y$-periodic, $Y=\left(y_{1 R}, y_{1 L}\right) \times\left(y_{2 R}, y_{2 L}\right)$. The tensor $E_{i j k l}^{\varepsilon}$ of material constants satisfies the symmetry condition

$$
E_{i j k l}^{\varepsilon}=E_{j i k l}^{\varepsilon}=E_{i j l k}^{\varepsilon}=E_{k l i j}^{\varepsilon} .
$$


It is also assumed that $E_{i j k l}^{\varepsilon}$ satisfies the strong ellipticity condition for every $\boldsymbol{x}$

$$
E_{i j k l}^{\varepsilon}(x) X_{i j} X_{k l} \geqslant m X_{i j} X_{i j} \text { for some } m>0 \text { and for every } X_{i j}=X_{j i}
$$

for every $\varepsilon$; here the Einstein summation convention is applied. Let the domain occupied by a structure be denoted by $\Omega$, with a Lipschitz boundary $\Gamma$, and let a body force $f$ be applied in $\Omega$. Let a traction $t$ be applied along a part of the boundary $\Gamma_{\mathrm{T}}$ which is the complement of the part of the boundary $\Gamma_{\mathrm{D}}$ on which displacement components are specified, see Fig. 1 .

In this setting, equilibrium of the structure can be characterized by the minimum potential

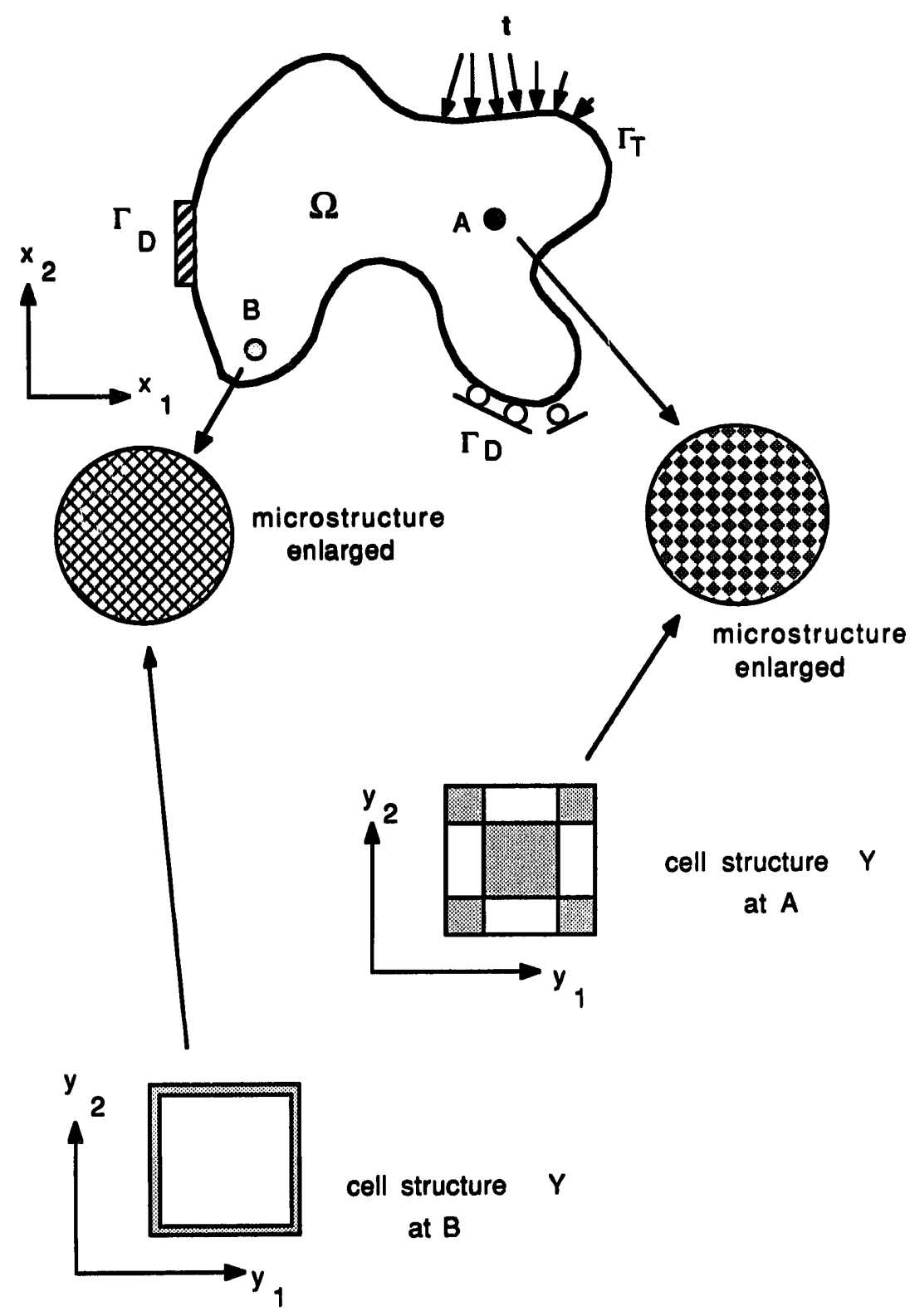

Fig. 1. A structure with composite microstructure. 
energy principle: the displacement $u^{\varepsilon}$ for equilibrium is the solution of the minimization problem

$$
\min _{\boldsymbol{v}^{\varepsilon} \in U} F^{\varepsilon}\left(\boldsymbol{v}^{\varepsilon}\right),
$$

where $F^{\varepsilon}$ is the total potential energy defined by

$$
\begin{aligned}
& F^{\varepsilon}\left(v^{\varepsilon}\right)=\frac{1}{2} a^{\varepsilon}\left(v^{\varepsilon}, v^{\varepsilon}\right)-L\left(v^{\varepsilon}\right), \\
& a^{\varepsilon}(u, v)=\int_{\Omega} E_{i j k l}^{\varepsilon} \varepsilon_{k l}(u) \varepsilon_{i j}(v) \mathrm{d} x, \\
& L(v)=\int_{\Omega} f \cdot v \mathrm{~d} x+\int_{\Gamma_{\mathrm{T}}} t \cdot v \mathrm{~d} s,
\end{aligned}
$$

and $U$ is the admissible linear manifold such that

$$
U=\left\{v=v_{i} e_{i}: v_{i} \in H^{1}(\Omega), v_{i}=g_{i} \text { on } \Gamma_{\mathrm{D}}\right\} .
$$

Here, $g$ is the specified displacement along the boundary $\Gamma_{D}$, and $\varepsilon(v)$ is the linearized strain tensor. It is clear that the solution to the minimum problem depends on the parameter $\varepsilon$ characterizing the microstructure, i.e.,

$$
u^{\varepsilon}(x)=u(x, y), \quad y=x / \varepsilon .
$$

Now assume that an arbitrary admissible displacement $v^{8}$ is expanded as

$$
v^{\varepsilon}(x)=v_{0}(x)+\varepsilon v_{1}(x, y), \quad y=x / \varepsilon,
$$

where $v_{0} \in U$ and $v_{1}(x, y)$ is defined in $\Omega \times Y, v_{1}(x,$.$) is Y$-periodic, and $v_{1}=0$ on $\Gamma_{D} \times Y$. Noting that

$$
\frac{\partial}{\partial x_{i}}\left(\left.\varphi(x, y)\right|_{y=x / \varepsilon}\right)=\frac{\partial \varphi}{\partial x_{i}}+\frac{1}{\varepsilon} \frac{\partial \varphi}{\partial y_{i}},
$$

the total potential energy is expanded as

$$
\begin{aligned}
F^{\varepsilon}\left(v^{\varepsilon}\right)= & \frac{1}{2} \int_{\Omega} E_{i j k l}^{\varepsilon}\left(\frac{\partial v_{0 k}}{\partial x_{l}}+\frac{\partial v_{1 k}}{\partial y_{l}}\right)\left(\frac{\partial v_{0 i}}{\partial x_{j}}+\frac{\partial v_{1 i}}{\partial y_{j}}\right) \mathrm{d} x \\
& -\int_{\Omega} f \cdot v_{0} \mathrm{~d} x-\int_{\Gamma_{\mathrm{T}}} t \cdot v_{0} \mathrm{~d} s+\varepsilon R^{\varepsilon}\left(v_{0}, v_{1}\right),
\end{aligned}
$$

where $R^{\varepsilon}$ is the remainder which is bounded for fixed $v_{0}$ and $v_{1}$. Applying the relation 


$$
\lim _{\varepsilon \rightarrow 0} \int_{\Omega} \Phi(x, x / \varepsilon) \mathrm{d} x=\frac{1}{|Y|} \int_{\Omega} \int_{Y} \Phi(x, y) \mathrm{d} y \mathrm{~d} x,
$$

we see that

where

$$
\begin{aligned}
\lim _{\varepsilon \rightarrow 0} F^{\varepsilon}\left(v^{\varepsilon}\right)= & F\left(v_{0}, v_{1}\right), \\
F\left(v_{0}, v_{1}\right)= & \frac{1}{|Y|} \frac{1}{2} \int_{\Omega} \int_{Y} E_{i j k l}(x, y)\left(\frac{\partial v_{0 k}}{\partial x_{i}}+\frac{\partial v_{1 k}}{\partial y_{l}}\right)\left(\frac{\partial v_{0 i}}{\partial x_{j}}+\frac{\partial v_{1 i}}{\partial y_{j}}\right) \mathrm{d} y \mathrm{~d} x \\
& -\int_{\Omega} f \cdot v_{0} \mathrm{~d} x-\int_{\Gamma_{\mathrm{T}}} t \cdot v_{0} \mathrm{~d} s .
\end{aligned}
$$

If the pair $\left\{u_{0}, u_{1}\right\}$ is the minimizer of the functional $F$, it satisfies the following two equations:

and

$$
\begin{gathered}
\frac{1}{|Y|} \int_{\Omega} \int_{Y} E_{i j k l}(x, y)\left(\frac{\partial u_{0 k}}{\partial x_{l}}+\frac{\partial u_{1 k}}{\partial y_{l}}\right) \frac{\partial v_{0 i}}{\partial x_{j}} \mathrm{~d} y \mathrm{~d} x \\
=\int_{\Omega} f \cdot v_{0} \mathrm{~d} x+\int_{\Gamma_{\mathrm{T}}} t \cdot v_{0} \mathrm{~d} s \text { for every } v_{0},
\end{gathered}
$$

$$
\frac{1}{|Y|} \int_{\Omega} \int_{Y} E_{i j k l}(x, y)\left(\frac{\partial u_{0 k}}{\partial x_{l}}+\frac{\partial u_{1 k}}{\partial y_{l}}\right) \frac{\partial v_{1 i}}{\partial x_{j}} \mathrm{~d} y \mathrm{~d} x=0 \text { for every } v_{1} \text {. }
$$

If $u_{1}$ is assumed to be decomposed into

$$
u_{1 k}(x, y)=-\chi_{k}^{p q}(y) \frac{\partial u_{0 p}}{\partial x_{q}}(x),
$$

and if $\chi^{p q}$ satisfies

$$
\int_{Y}\left(E_{i j k l}-E_{i j p q} \frac{\partial \chi_{p}^{k l}}{\partial y_{q}}\right) \frac{\partial v_{1 i}}{\partial y_{j}} \mathrm{~d} y=0 \text { for } k, l=1 \text { and } 2,
$$

the second equation is automatically satisfied. Substitution of (28) into the first equation yields the homogenized equation

where

$$
\int_{\Omega} E_{i j k l}^{H}(x) \frac{\partial u_{0 k}}{\partial x_{l}} \frac{\partial v_{0 i}}{\partial x_{j}} \mathrm{~d} x=\int_{\Omega} f \cdot v_{0} \mathrm{~d} x+\int_{\Gamma_{\mathrm{T}}} t \cdot v_{0} \mathrm{~d} s \text { for every } v_{0},
$$

Define

$$
E_{i j k l}^{H}(x)=\frac{1}{|Y|} \int_{Y}\left(E_{i j k l}(x, y)-E_{i j p q}(x, y) \frac{\partial \chi_{p}^{k l}}{\partial y_{q}}\right) \mathrm{d} y .
$$

$$
a_{H}(u, v)=\int_{\Omega} E_{i j k l}^{H}(x) \frac{\partial u_{k}}{\partial x_{l}} \frac{\partial v_{i}}{\partial x_{j}} \mathrm{j} x
$$




$$
a_{Y}\left(\chi^{k l}, v\right)=\int_{Y} E_{i j p q}(x, y) \frac{\partial \chi_{p}^{k l}}{\partial y_{q}} \frac{\partial v_{i}}{\partial y_{j}} \mathrm{~d} y
$$

and

$$
L_{Y}^{k l}(\boldsymbol{v})=\int_{Y} E_{i j k l} \frac{\partial v_{i}}{\partial y_{j}} \mathrm{~d} y .
$$

Thus, the following problem on the microrsopic level and associated homogenized problem on the macroscopic level constitute the necessary (and sufficient) conditions that are obtained by passing to the limit $\varepsilon \rightarrow 0$ in the minimum principle (24).

Problem at microscopic level in the cell $Y$ :

$$
\chi^{k l} \in U_{Y}: \quad a_{Y}\left(\chi^{k l}, v\right)=L_{Y}^{k i}(v) \quad \text { for every } v \in U_{Y},
$$

where $U_{Y}$ is the admissible space defined in the cell $Y$ :

$$
U_{Y}=\left\{v=v_{i} e_{Y_{i}}: v_{i} \in H^{1}(Y) / R, v_{i} \text { takes equal values on opposite faces of } Y\right\}
$$

( $R$ is the set of constant functions defined on $Y$ ).

Problem at macroscopic level: the homogenized problem on $\Omega$ :

$$
u \in U: a_{H}(u, v)=L(v) \text { for every } v \in U_{0},
$$

where $U_{0}$ is the homogeneous case of $U$, i.e., $g=0$.

It is noted that the homogenized elasticity tensor is also given by

$$
E_{i j k l}^{H}(x)=\frac{1}{|Y|} a_{Y}\left(\chi^{k l}-P^{k l}, \chi^{i j}-P^{i j}\right),
$$

where $P^{i j}=y_{i} \delta_{i k} e_{Y k}$. Also note that the homogenized energy form $a_{H}(u, v)$ is given by the minimization problem

$$
a_{H}(u, v)=\lim _{\varepsilon \rightarrow 0} \min _{\varphi \in U Y} \frac{1}{|Y|} a^{\varepsilon}(u+\varphi, v+\varphi),
$$

where the average energy in the unı cell is minimized over all periodic disturbance deformation fields $\varphi$ of period $Y$ (cf. [35]).

We note here that the derivation above is for a microstructure without holes, but the formulas for the effective material constants still holds true in this case also, as proved in $[45,46]$.

For shape optimization, it is necessary to solve the cell problem to determine the homogenized elasticity tensor for a given microstructure that can define an appropriate material distribution. Thus, the cell problem is solved by finite element methods. To do this, the cell domain $Y$ is discretized by appropriate finite elements, the admissible space $U_{Y}$ is approximated by $U_{Y h}$ using a finite element method, and then the finite element approxima- 
tion $\chi_{h}^{k l} \in U_{Y h}$ of the $\chi^{k l}$ is obtained as the solution of the discrete problem

$$
\chi_{h}^{k l} \in U_{Y h}: a_{Y}\left(\chi_{h}^{k l}, v_{h}\right)=L_{Y}^{k l}\left(v_{h}\right) \text { for every } v_{h} \in U_{Y h} .
$$

Using the approximation the homogenized elasticity tensor is defined by

$$
{ }_{h} E_{i j k l}^{H}(x)=\frac{1}{|Y|} \int_{Y}\left(E_{i j k l}(x, y)-E_{i j p q}(x, y) \frac{\partial \chi_{h p}^{k l}}{\partial y_{q}}\right) \mathrm{d} y .
$$

By simple algebra, it can be obtained that

$$
{ }_{h} E_{i j k l}^{H}-E_{i j k l}^{H}=\frac{1}{|Y|} a_{Y}\left(\chi_{h}^{k l}-\chi^{k l}, \chi_{h}^{i j}-\chi^{i j}\right) \text {. }
$$

Noting that the orthogonality of the approximation error to the finite element space implies that

$$
a_{Y}\left(\chi_{h}^{k l}-\chi^{k l}, \chi_{h}^{i j}-\chi^{i j}\right)=a_{Y}\left(\chi_{h}^{k l}-\chi^{k l}, v_{h}-\chi^{i j}\right) \text { for every } v_{h} \in U_{Y h},
$$

the finite element approximation error of the homogenized elasticity tensor is bounded by the interpolation error of $\chi^{i j}$ and $\chi^{k l}$ :

$$
\left.\right|_{h} E_{i j k l}^{H}-E_{i j k l}^{H} \mid \leqslant \frac{1}{|Y|} \sqrt{a_{Y}\left(\chi_{1}^{i j}-\chi^{i j}, \chi_{1}^{i j}-\chi^{i j}\right)} \sqrt{a_{Y}\left(\chi_{1}^{k l}-\chi^{k l}, \chi_{1}^{k l}-\chi^{k l}\right)},
$$

where $\chi_{\mathrm{I}}^{i j}$ is the finite element interpolation of $\chi^{i j}$.

Only plane problems are treated in this paper, so we let the cell structure $Y$ be discretized by four-node isoparametric elements in which each component of $\chi^{k l}$ for $k, j=1$ and 2 is interpolated by bilinear polynomials of the coordinates in the master element.

The finite element calculations on the unit cell are used for the computation of the homogenized elasticity tensor which is applied for the shape optimization. An important issue in the finite element modeling of the cell structure is thus whether the results are very sensitive with respect to the specific choice of finite element mesh. To examine mesh sensitivity, consider two different microstructures as shown in Fig. 2. The first one consists of two

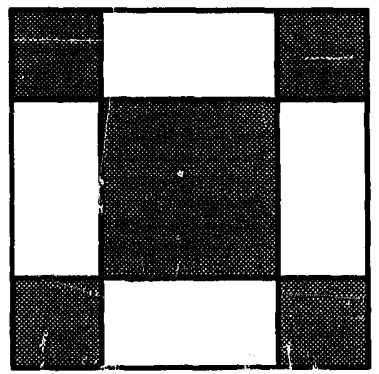

First Cell Structure (hard/soft materials)

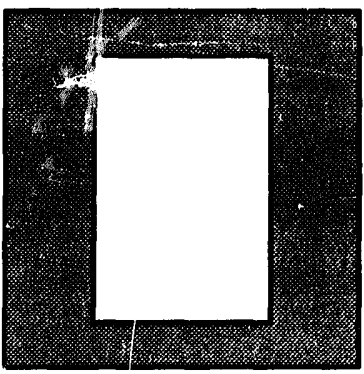

second cell structure (hole/substance)

Fig. 2. Two different microstructures. 
different materials; a soft and a hard material. If Young's modulus of the soft material is very small, say $10^{-2}-10^{-3}$ times the Young's modulus of the hard material, the soft part can be regarded as voids or holes. The second cell contains a rectangular hole. That is, the hole is not approximated by a very soft material in the second case.

For the first cell, the soft and hard materials are isotropic, and are characterized by Young's moduli $E_{\text {soft }}=10$ and $E_{\text {hard }}=1000$ ard the same Poisson's ratio $\nu=0.3$. In order to test for mesh sensitivity, the initial discretization is defined by $16 \times 16$ uniform, square four-node isuparametric elements. On this finite element model the $h$-adaptive method is then applied twice, using an error measure based on the interpolation error as given above (for details, see [45]). The resulting finite element meshes are sh vn in Fig. 3 , and the computed homogenized elasticity tensors are given in Table 1 . Note that plane stress is assumed in this study. There is a difference of about $20 \%$ between the values of $E_{1111}^{H}$ obtained from use of the initial and adapted meshes, respectively. It is thus clear that the mesh sensitivity is very significant in this case.

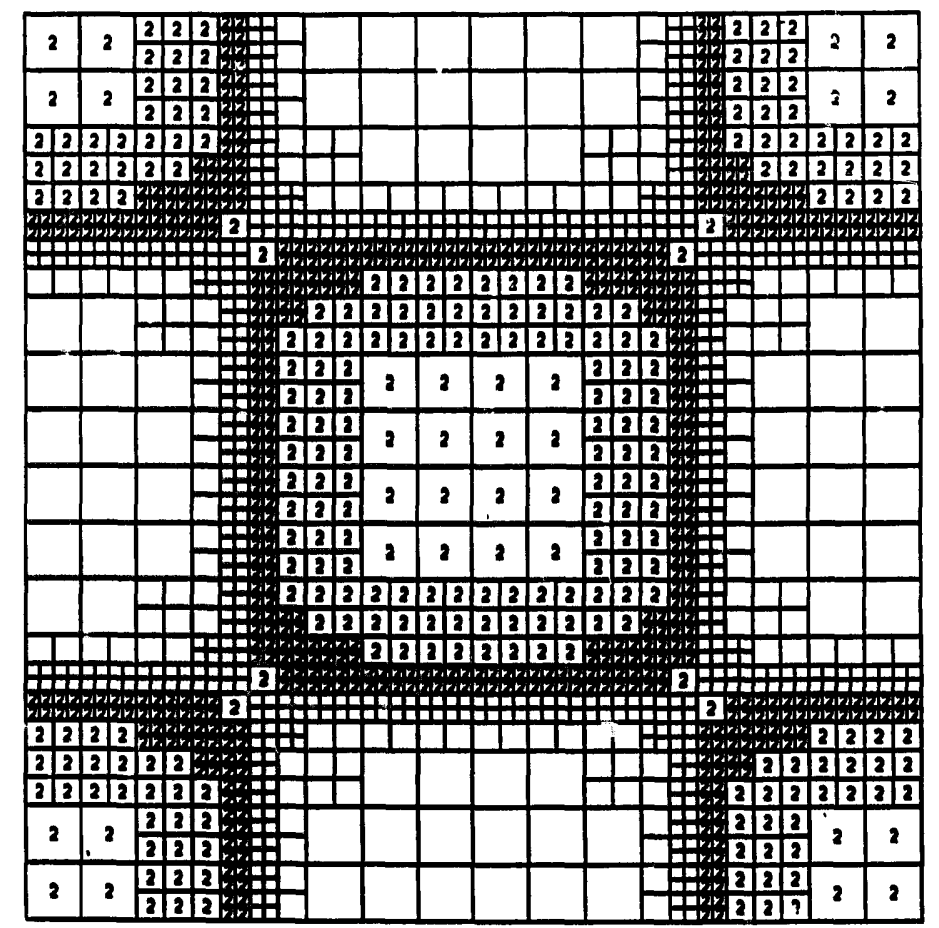

Fig. 3. Adapted finite element mesh (cell 1).

Table 1

Finite element mesh sensitivity for the first cell

\begin{tabular}{ccccc}
\hline Mesh & $E_{1111}$ & $E_{1122}$ & $E_{2222}$ & $E_{1212}$ \\
\hline $16 \times 16$ & 149.80 & 71.61 & 149.80 & 87.12 \\
1st Adap & 127.12 & 62.91 & 127.12 & 75.90 \\
2nd Adap & 125.79 & $6: .62$ & 125.79 & 75.28 \\
\hline
\end{tabular}


For the second cell, which has a rectangular hole, a $20 \times 20$ mesh is used at the initial discretization, and the $h$-adaptation is applied three times as shown in Fig. 4 . In this case, the substance material is characterized by $E_{1111}=E_{2222}=30$ and $E_{1122}=E_{1212}=10$. After homogenization is performed, the computed elasticity tensors are as given in Table 2 . In this case, mesh sensitivity is very small, say, less than $1.5 \%$ in $E_{1111}$.

From the above examples it can be concluded that it is better not to replace holes by soft materials when the goal is to determine the homogenized elasticity tensor for materials containing microscale voids. Thus, in the present study, a cell structure with a rectangular hole is considered.

For use in shape optimization, the homogenized elasticity tensor must be obtained for a "continuously" varying size of a rectangular hole in the cell so that the density of the substance of materials can be a continuous design variable for the optimal "material distribution." To this end, it is certainly impossible to determine the homogenized elastic

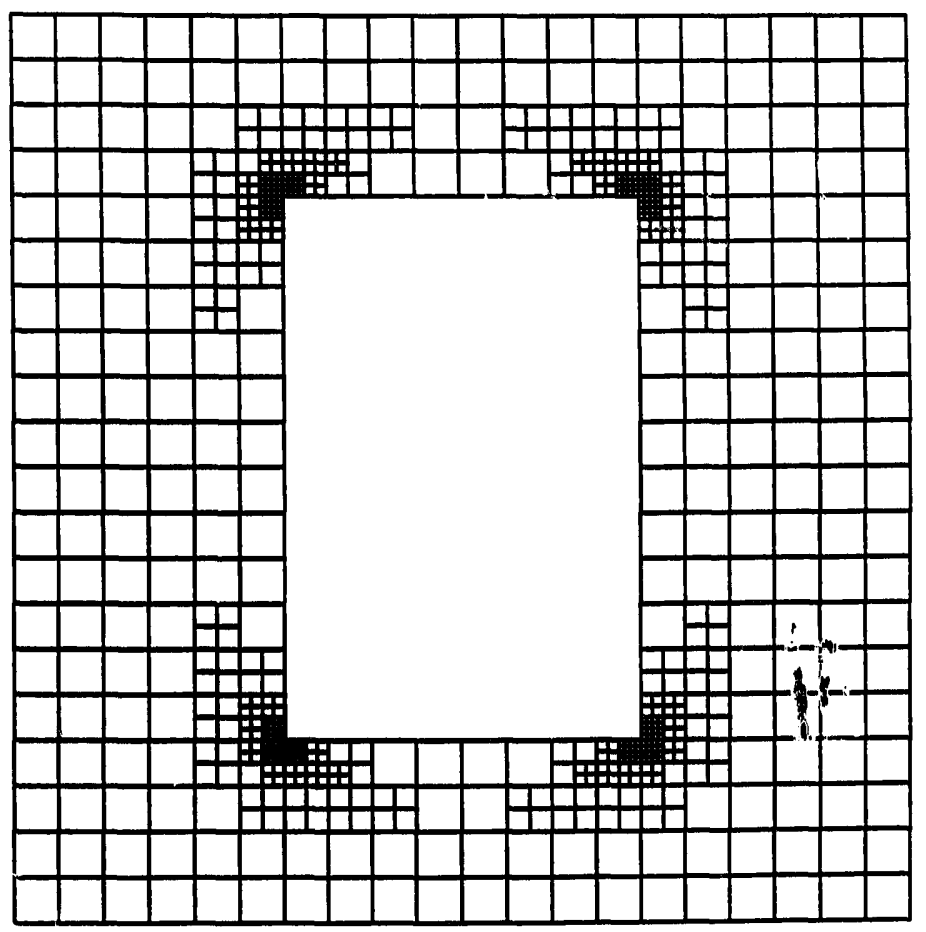

Fig. 4. Adapted finite element grid (cell 2).

Table 2

Finite element mesh sensitivity for the second cell

\begin{tabular}{ccccc}
\hline Mesh & $E_{1111}$ & $E_{1122}$ & $E_{2222}$ & $E_{1212}$ \\
\hline Init & 13.015 & 3.241 & 17.552 & 2.785 \\
1st Adap & 12.910 & 3.178 & 17.473 & 2.714 \\
2nd Adap & 12.865 & 3.146 & 17.437 & 2.683 \\
3rd Adap & 12.844 & 3.131 & 17.421 & 2.668 \\
\hline
\end{tabular}




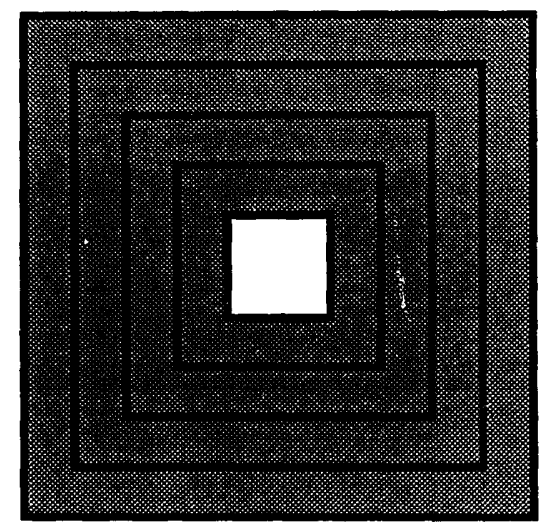

Fig. 5. Representative square holes in the unit (square) cell.

constant for all the possibilities of the material density (that is, the size of a rectangular hole in the cell). Thus, we compute the homogenized elastic tensors for certain sizes of the hole, and other values are then interpoiated by, e.g., Legendre polynomials, in order to have a continuous variation with respect to material density. An example for a square hole is shown in Figs. 5 and 6 in which four representative square holes and the interpolated components of the homogenized elastic tensor are given, respectively. It is noted that the variation of the elastic moduli is highly nonlinear, as shown in Fig. 6. This nonlinearity win' play an important role in the shape optimization and will be examined by comparing the res. its obtained in the case of a linearly varying elasticity tensor. It is also important to note that if the cell structure is rotated in relation to a fixed reference frame (for example the coordinate system of the shape optimization problem), the transformed elastic tensor is very different from the one for the unrotated case. The influence of the rotation of the cell becomes very strong when the size of the hole becomes large, as shown in Fig. 7, and it can be seen that a possibility of rotation

Algidity as Function of Dansity (equere holo)

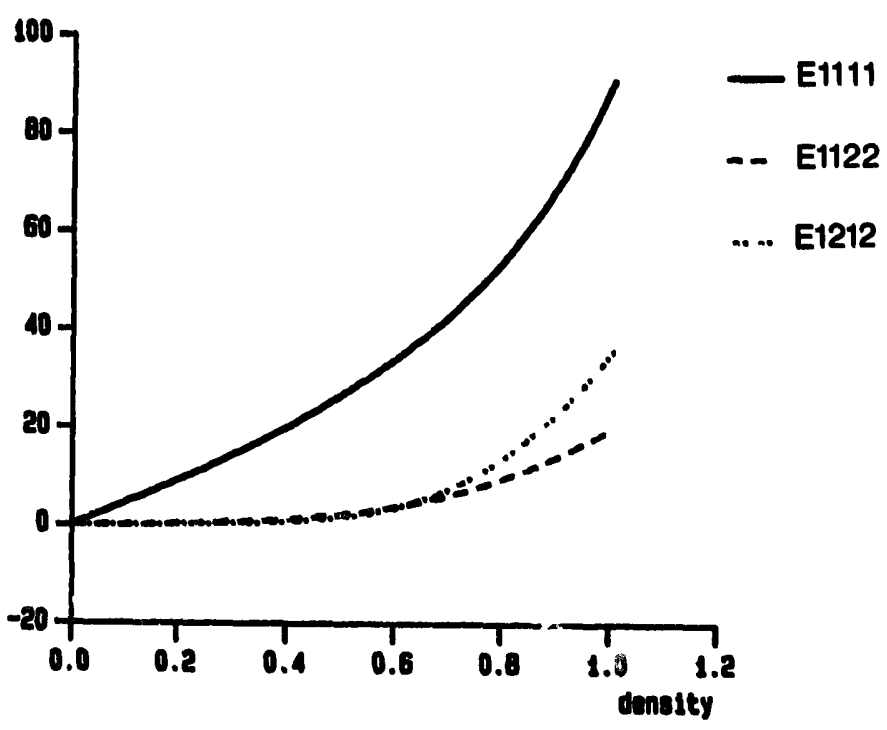

Fig. 6. Interpolated elastic moduli. 


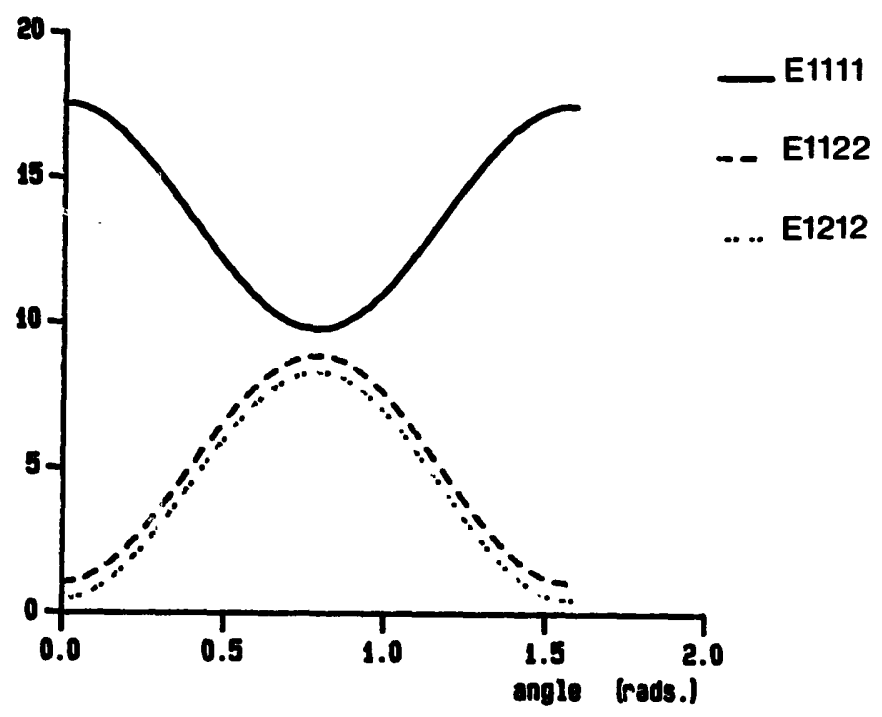

Fig. 7. Elastic moduli with respect to rotation of the cell.

of the cell is quite important for our purpose of shape optimization. This strong dependence on rotation is clear from a physical point of view, as a cell with a large hole will have almost no shearing stiffness, while the same cell rotated 45 degrees has a quite significant shearing stiffness.

\section{The optimization method}

The method of homogenization allows us to compute the effective properties for a material with infinitely many, infinitely small holes and we are thus in a position to compute the optimal distribution of such a composite material in a given domain. Let $\Omega$ be a suitable chosen domain in $\mathbb{R}^{2}$ that allows you to introduce the given surface tractions (see Fig. 8). Assume that a design composite with a square unit cell with a square hole is to be distributed in this reference domain $\boldsymbol{\Omega}$. Homogenization gives us a relationship

$$
E_{i j k l}=E_{i j k l}(\alpha, \theta)
$$

between the effective material constants of the composite, the pointwise varying hole size $\alpha$, and the pointwise varying angle of rotation $\theta$ of the pell. The hole size $\alpha$ is given as $\alpha=A^{2}$, with $A$ being the length of the side of the hole. The density of material is $\mu=(1-\alpha)$.

The problem formulation for minimization of compliance for a given volume of material by distribution of the composite material takes on the form

$\underset{\mu, \theta}{\operatorname{minimize}} L(u)$

so that $a(u, v)=L(v)$ for all $v \in U$,

$\int_{\Omega} \mu \mathrm{d} x \leqslant$ Vol , $0 \leqslant \mu \leqslant 1$, 

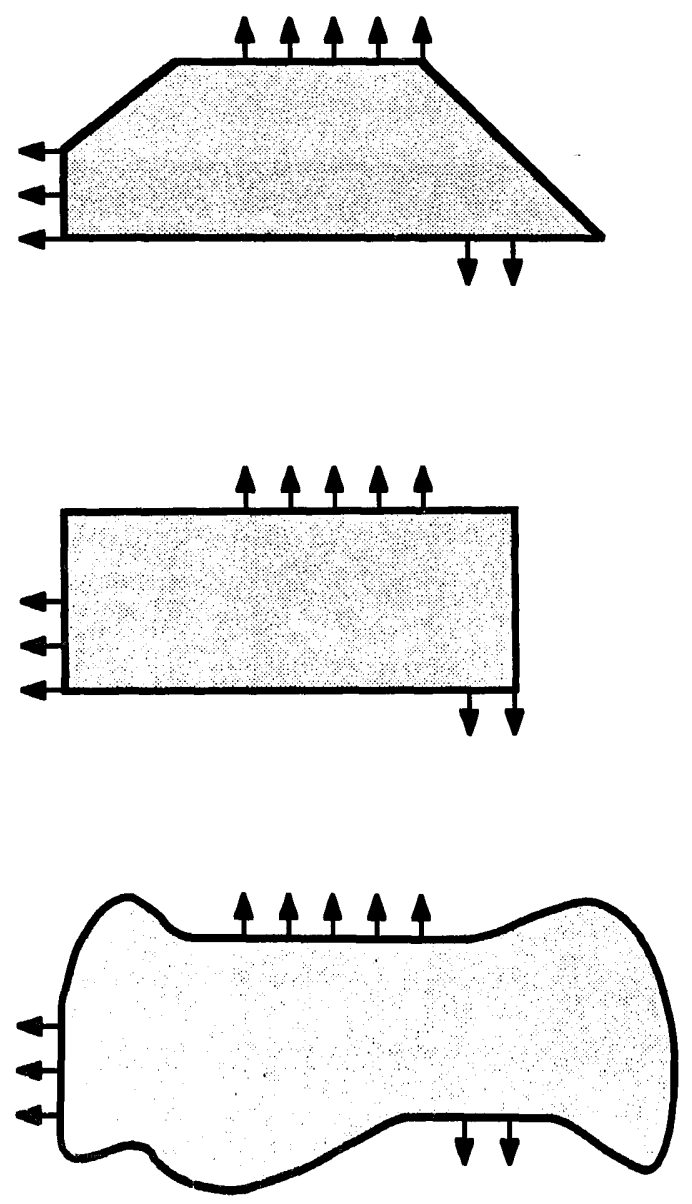

Fig. 8. Different choices of design domain $\boldsymbol{\Omega}$, for a given set of surface tractions.

where the notation of Section 2 is used. We notice that this problem finds the optimal distribution of square holes in the domain $\Omega$ and it is thus a shape optimization problem, even though it has the form of a sizing problem!

Solving problem (46) by means of computations is carried in the following three steps:

Step 1. The material constants $E_{i j k l}(\mu)$ are computed for a number of values of cell hole size (say 6 values) and the complete functional dependence of $E_{i j k l}$ on $\mu$ is approximated by interpolation with Legendre polynomials defined on the interval $[0,1]$. The dependence of $E_{i j k l}$ on $\theta$ is given by the well-known frame rotation formulas, as mentioned in Section 3.

Step 2. A finite element approximation scheme is constructed for the analysis problem of (46). $\Omega$ should thus be chosen in order to simplify the analysis problem as much as possible.

Step 3. The design variables $\mu$ and $\theta$ are discretized by assigning a constant value in each element of the finite element model. An optimization scheme based on the optimality criteria for (46) is constructed. This scheme requires data provided by the homogenization computations as well as the finite element analysis. 
The methodology for finding the optimality criteria for the problem stated above is well known [47], so we will restrict ourselves to stating the result. As the adjoint deformation of the problem is just the deformation field $u$, the optimality criteria are given as

$$
\begin{aligned}
& \frac{\partial E_{i j k l}}{\partial \mu} \varepsilon_{i j}(u) \varepsilon_{k l}(u)=\Lambda+\eta_{1}-\eta_{2}, \\
& \frac{\partial E_{i j k l}}{\partial \theta} \varepsilon_{i j}(u) \varepsilon_{k l}(u)=0,
\end{aligned}
$$

with $\Lambda \geqslant 0$ being the Lagrange multiplier associated with the volume constraint and $\eta_{i}, i=1$, $2, \eta_{i} \geqslant 0$, the Lagrange multipliers for the constraints $\mu-1 \leqslant 0$ and $\mu \leqslant 0$, respectively. The material parameters $E_{i j k l}$ are given explicitly in terms of Legendre polynomials in $\mu$ and in terms of cosines and sines in $\theta$. Thus the derivatives appearing in (47) and (48) can be computed explicitly. The strain terms of (47) and (48) are in their turn computed using the finite element approximation discussed above. Finally, the nonlinear equations (46) and (47) in $\mu$ and $\theta$ are solved by iteration using a recursion formula

$$
\mu_{k+1}= \begin{cases}\max \left\{(1-\zeta) \mu_{k}, 0\right\} & \text { if } h_{k} D_{k}^{\eta} \leqslant \max \left\{(1-\zeta) \mu_{k}, 0\right\} \\ \mu_{k} D_{k}^{\eta} & \text { if } \max \left\{(1-\zeta) \mu_{k}, 0\right\} \leqslant h_{k} D_{k}^{\eta} \leqslant \min \left\{(1+\zeta) \mu_{k}, 1\right\}, \\ \min \left\{(1+\zeta) \mu_{k}, 1\right\} & \text { if } \min \left\{(1+\zeta) \mu_{k}, 1\right\} \leqslant h_{k} D_{k}^{\eta},\end{cases}
$$

for the density $\mu$. Here $D$ denotes

$$
\Lambda^{-1} \frac{\partial E_{i j k l}}{\partial \mu} \varepsilon_{i j}(u) \varepsilon_{k l}(u)
$$

$\eta$ is a weighting factor, and $\zeta$ a move limit. After updating $\mu_{k}$ to $\mu_{k+1}$, the updated angle $\theta_{k+1}$ is computed by solving (48) using a combined Newton-bisection method. Note that $\mu_{k+1}$ depends on the present value of the Lagrange multiplier $\Lambda_{k}$, and that $\Lambda_{k}$ should be adjusted in an inner iteration loop in order to satisfy the volume constraint (this constraint is active). The values of $\eta$ and $\zeta$ are chosen by experiment, in order to obtain a suitable rapid and stable convergence of the scheme.

The optimization algorithm described above is just one among many possible choices that can be made. When deciding on use of a specific algorithm in the present situation it has to be taken into account that a very fine discretization mesh will have to be used if you seek prediction of the fine details of shape. Thus the algorithm employed should be able to handle a large number of variables, as well as requiring only a few function calls, which in this case means performing a finite element analysis.

\section{Computational results}

The optimization method described above has been tested in various ways on the much studied problem of optimal shape design of a fillet. This problem is, for example, treated in $[4,5,7,8,48,49,50]$. 
Two different settings of the fillet problem will be presented, allowing for different possibilities for connecting the surface tractions of the problem. Figure 9 illustrates the two cases considered. For Case A only part of the structure is free to be redesigned and in Case B the full connecting structure between the surface tractions can be freely designed. Case $A$ is the situation usually seen in the literature.

The example problems are treated as plane elasticity problems, so the results on homogenization described in Section 3 apply in the form stated there. The optimization problem that was solved for the examples was the case of minimization of compliance for a given volume of material, as described in Section 4. In what follows, results for three different volume constraints will be illustrated, the volume constraint being given as the percentage of the full design area that is available for the construction of the fillet.

For the sake of comparison, a design optimization was also carried out for the case of a rigidity that is linear in the design variable, as for variable thickness sheets, cf. Section 2 . Such design problems have been studied in detail in [51], and are very well-behaved problems. Figure 10 shows results for this type of problems and illustrates that this representation of stiffness gives a good indication of the two-dimensional optimal shape for high volume fractions, while for low volume fractions it is difficult to interpret the results so as to define a two-dimensional shape. This is, of course, not surprising as we are dealing with a true three-dimensional problem that just happens to have a two-dimensional model. That is the variable thickness is a hidden three-dimensional feature. However, the design of variable thickness sheets does indeed give well-defined shapes as three-dimensional structures.

The testing of the design method using composite materials has been carried out for various different formulations that all relate to the description of the method given in Section 4. Tw:s
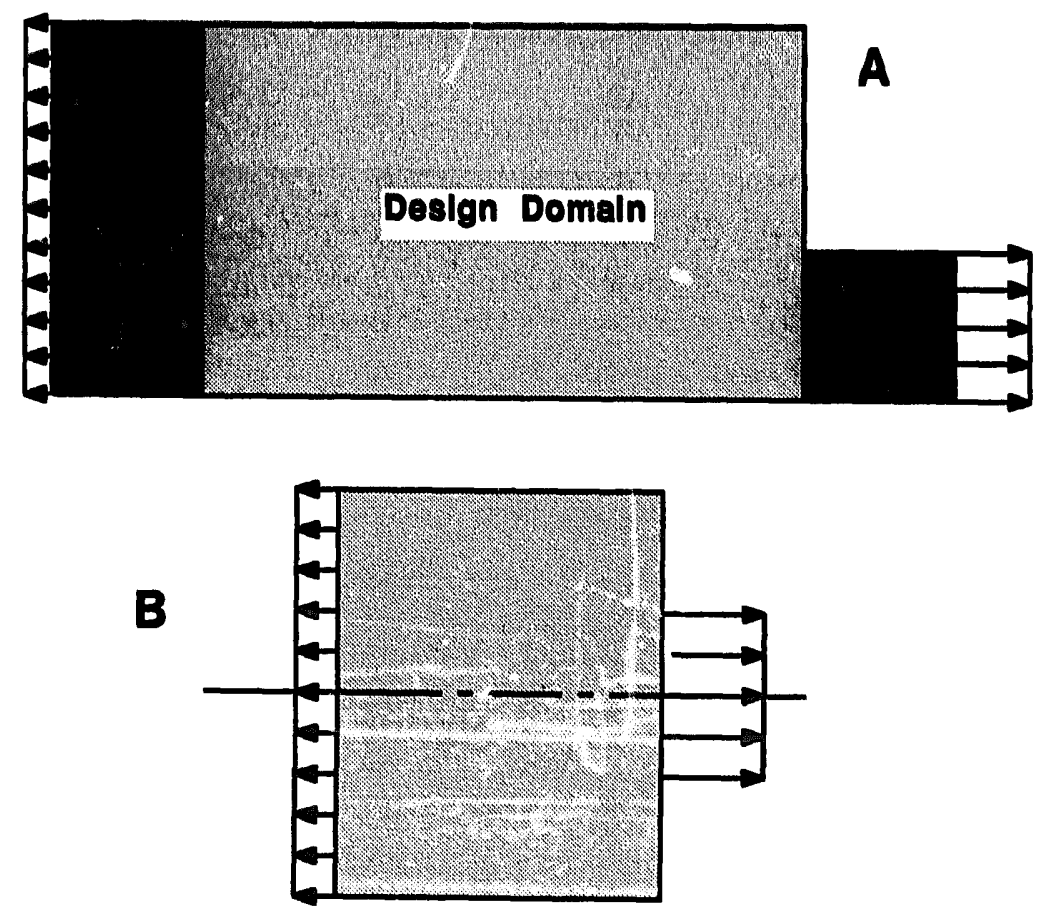

Fig. 9. The design cases. 

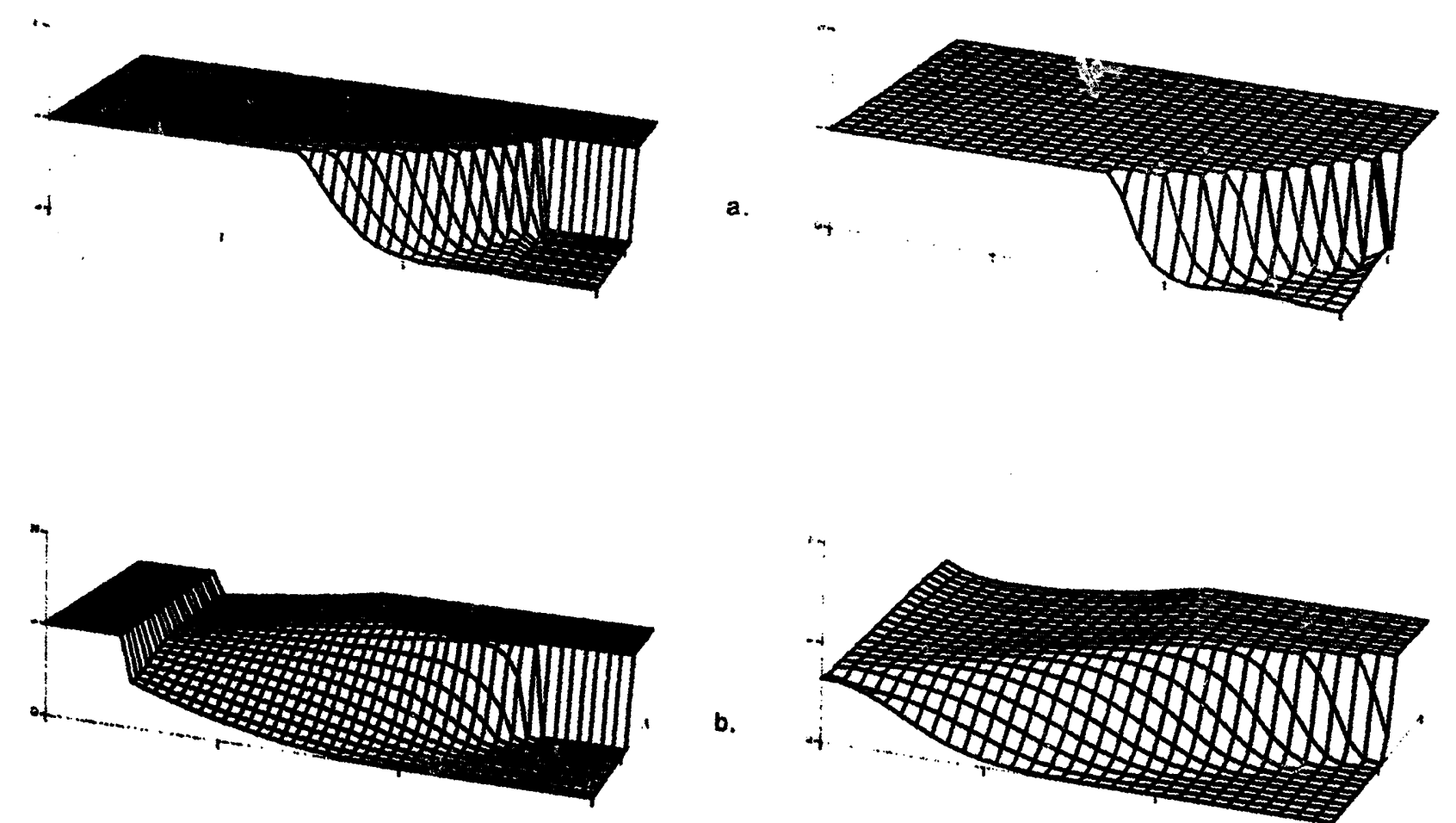

b.
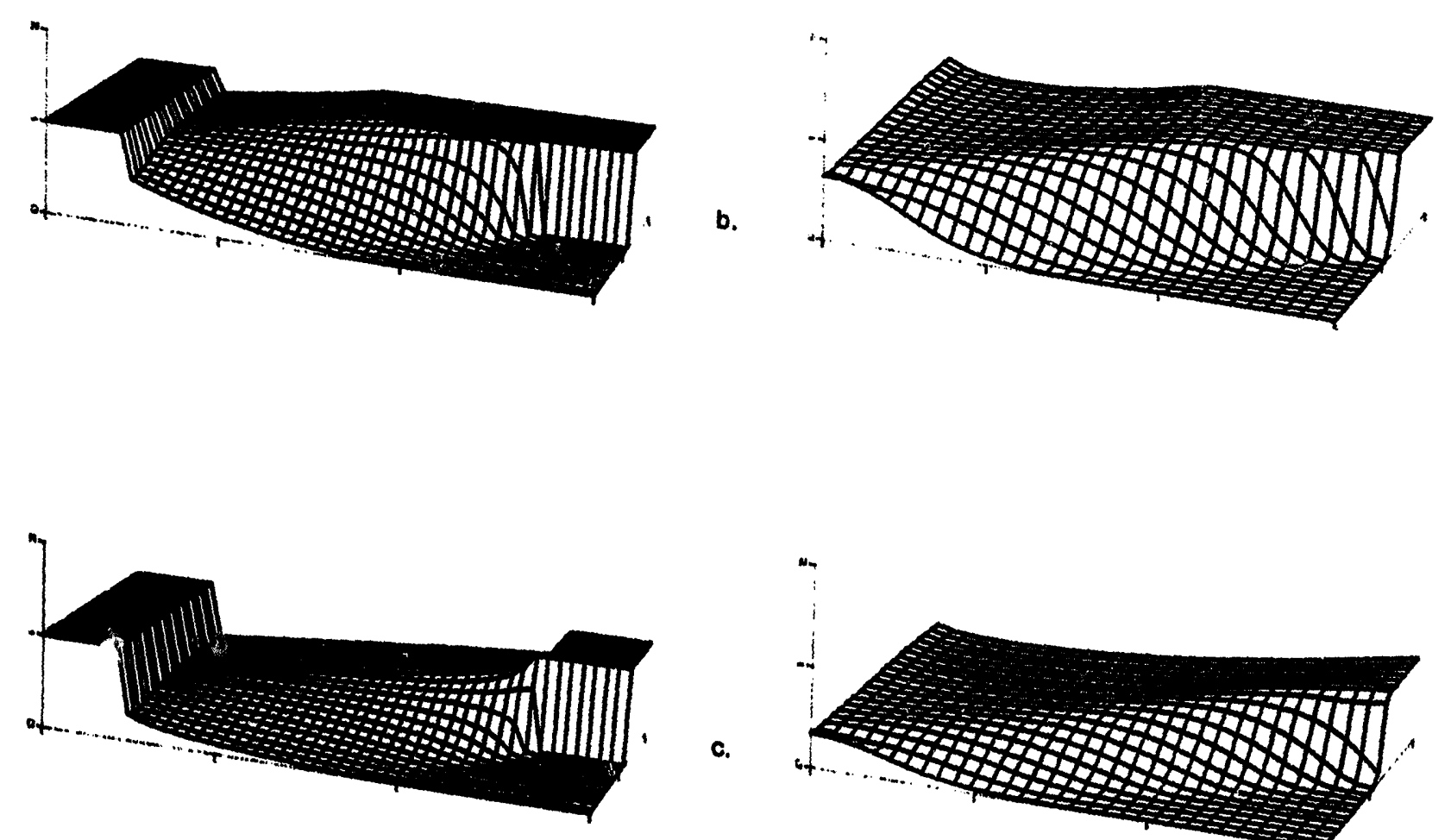

c.

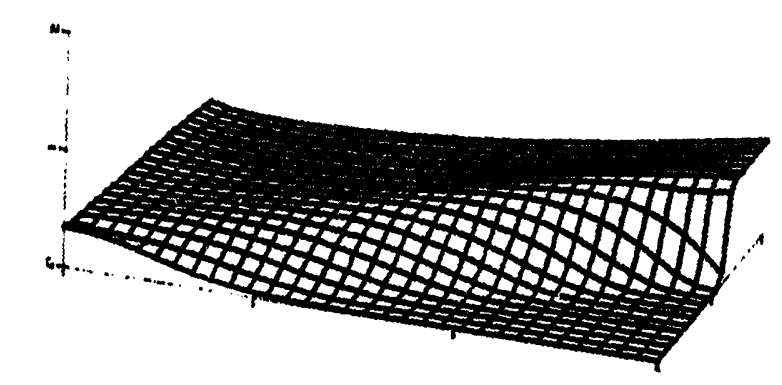

:ig. 10. Design of variable thickness sheets. Left-hand column for Case A, right-hand column for Case B. Volume is (a): $91 \%,(\mathrm{~b}): 64 \%,(\mathrm{c}): 36 \%$.

types of basic square cells were used, one with a square holes and just one variable needed to describe the resulting composite, and one with a rectangular hole that needs two design variables. For both types of composites an optimization was performed including or excluding the angle of cell rotation. Figures 11-14 and Table 3 show some of the results obtained.

A basic requirement for any shape design method is, of course, that the resulting designs are not very sensitive to the choice of finite element discretization, and that the results are 

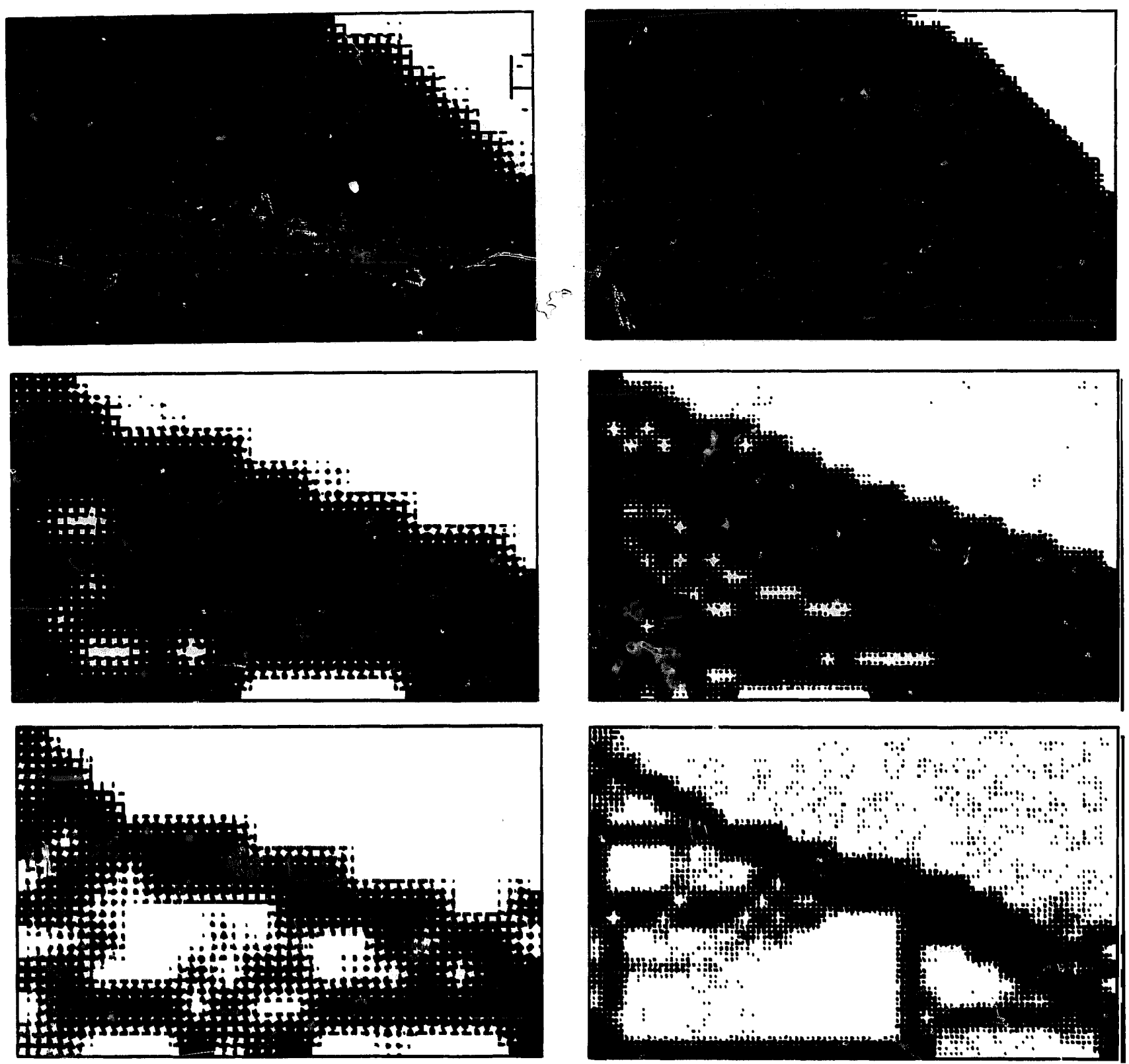

Fig. 11. Results for two different discretizations. Case B with voids as square holes. Left-hand column for $16 \times 10$ mesh, right-hand column for $32 \times 20$ mesh. Volume is $91 \%, 64 \%, 36 \%$, respectively. Black areas indicate material, and density of voids is illustrated as a removal of black areas. The voids are drawn element-wise as a rotated, macroscopic hole; note that the voids in reality are at microlevel.

stable with respect to an increase in the number of elements used. If both these requirements are satisfied it indicates that the optimization problem is well posed and one can have faith in the results. The present method seems to satisfy these requirements, as indicated in Fig. 11. Some caution should be taken when using the method with very fine discretizations. This may be tempting in order to get very good approximations of an eventual smooth design boundary, but a very fine mesh has a tendency to result in very small sensitivity of the functional near the 

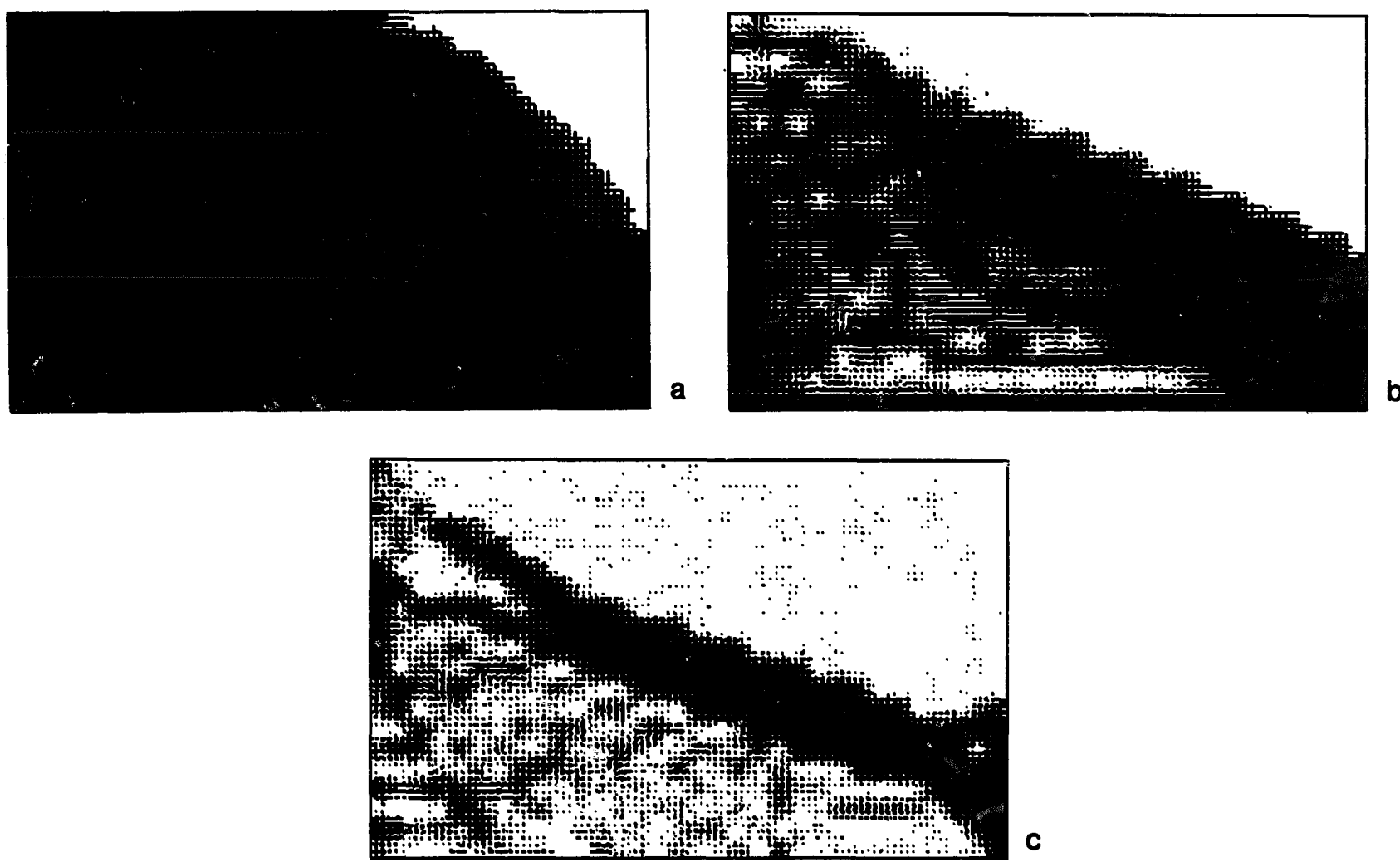

Fig. 12. Comparison with two-variable composite design. Case B with voids as rectangular holes. Volumes are (a): $91 \%$, (b): $64 \%$, (c): $36 \%$.

optimal design with respect to the design variables that are assigned values in each element. This has the effect that in such cases the design optimization becomes unreliable, and very sensitive to stoppping criteria, weighting factors, etc. However, for very little available material, a fine mesh is needed in order to be able to represent a beam-like layout of the material. As note $\lrcorner$ above the optimization was tested for square holes as well as rectangular holes in the square cell (see Fig. 12). The numerical experiments indicate that it is advantageous to use just one variable for the definition of the distribution of material. First oi all the introduction of more than one variable does not significantly improve the performance of the structure, so it does not pay off to use the extra computer time required in this case, and secondly the sensitivity of the compliance functional with respect to a too detailed description of the microstructure is so insignificant that the performance of the optimization scheme is degraded in a way similar to the degradation seen from use of a too fine discretization of the structure. The critical variable for the optimization method thus seems to be the density of material, and not the microscopic definition of this density. However, the different descriptions of the microstructure do lead to somewhat different structures, that is, the outer shapes are very similar while the inner structure (formation of microscopic holes, etc.) tend to differ. It should be pointed out that in any case the results give a fair definition of shape with very little true composite material in the optimal structure. Also, for moderately low volume fractions the method works like a method for lay-out of truss-like structures (see e.g. [52]). 

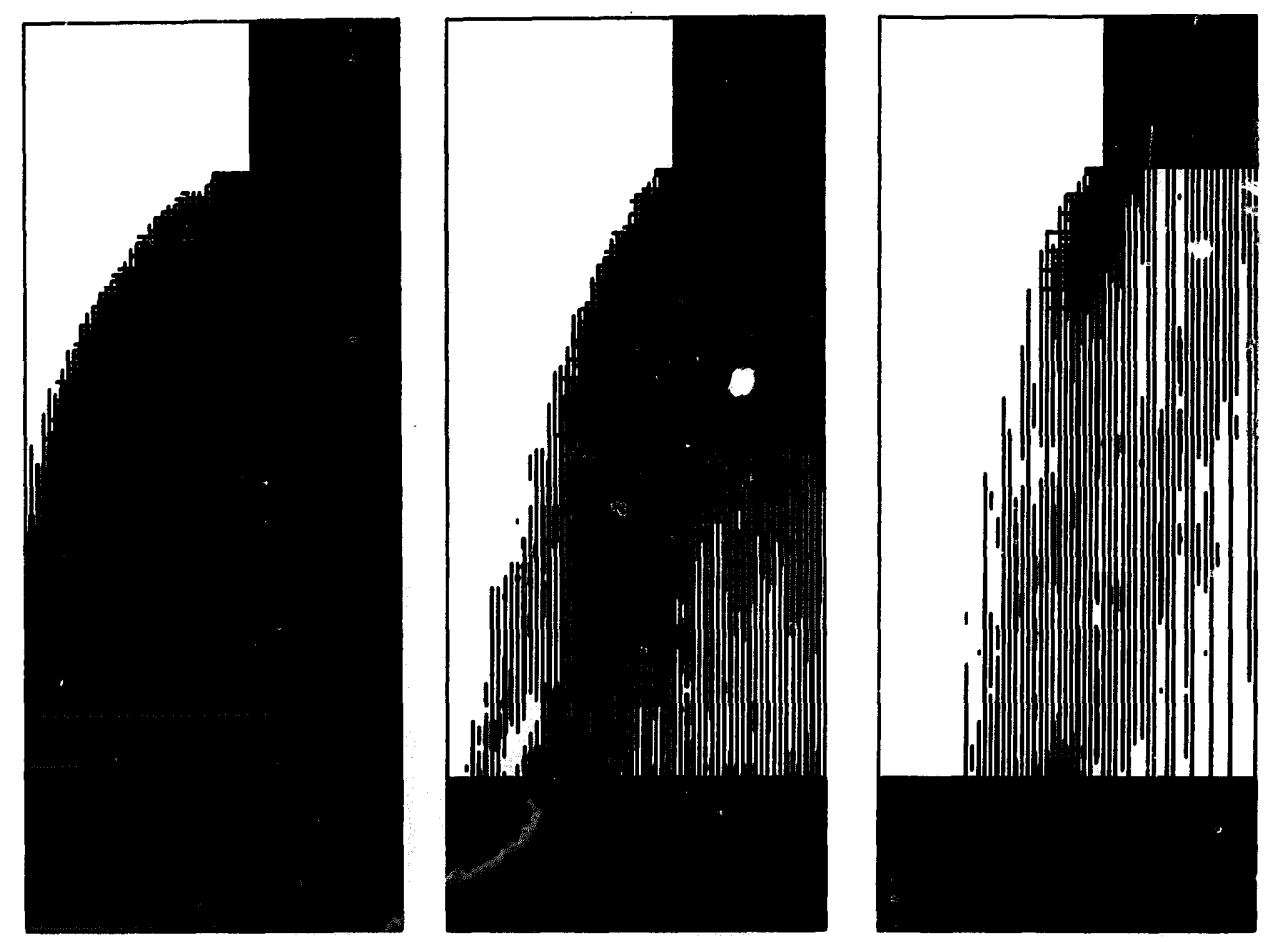

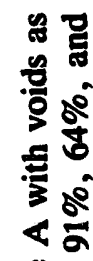

苋 产

0

롤
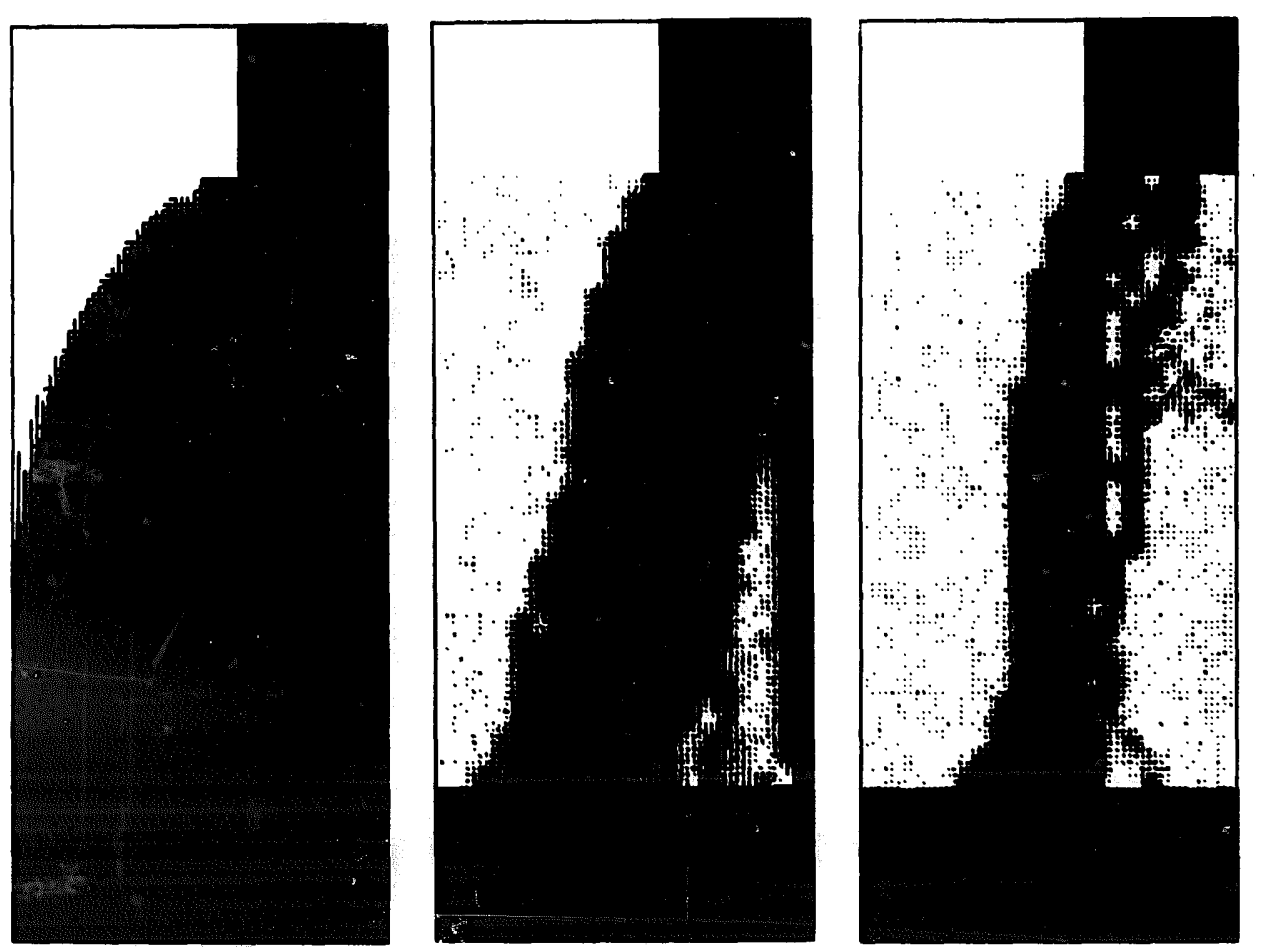

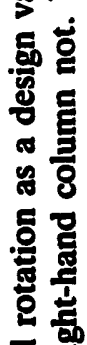

$\overline{8}$

造

节范

온 응

을

등

旁

요

륶통

คㅇ

늘 몰

氙宫

on

혈

을 응

它

믈

洁 ๓ 

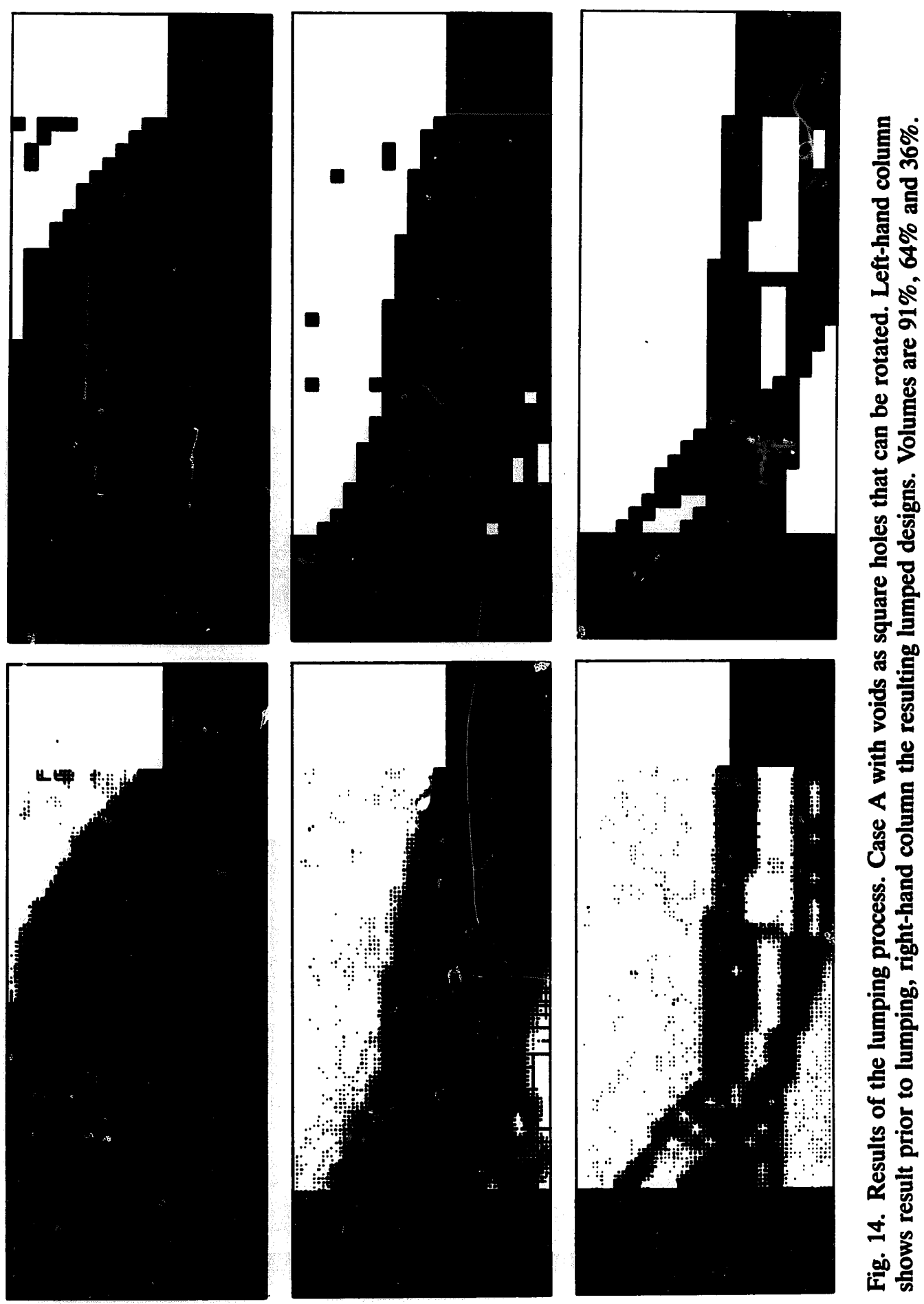

䙳

象

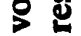

乎

要

틀

\%

을

送

छ

을 햄

o.

些0

昱朂

롱

농

을 을

象

$\dot{4}$

$\pm$

禹 
Table 3

Compliance values for optimal designs, Case A

\begin{tabular}{llll}
\hline & \multicolumn{3}{c}{ Volume } \\
\cline { 2 - 4 } Solid design area has & $91 \%$ & $64 \%$ & $36 \%$ \\
\hline square holes, coarse mesh & 3.53 & 4.19 & 7.55 \\
square holes, fine mesh & 3.54 & 4.26 & 7.96 \\
rectangular holes & 3.55 & 4.15 & 7.17 \\
rectangular holes, no rotation & 3.55 & 4.21 & 6.96 \\
rectangular holes, lumped & 3.55 & 4.17 & 7.27 \\
boundary variation & 3.55 & 4.20 & 8.96 \\
\hline
\end{tabular}

As discussed in the preceeding section the angle of rotation of the cell is important for the shearing stiffness of the resulting composite and it turns out to be very important for the optimization method presented here. It can be seen from Figs. 11-14 that the method gives a fairly precise definition of shape, that is, the optimization results in design with a density of material that typically only takes on the values 1 and 0 . Thus, even though intermediate densities are allowed, the optimization method builds a structure with very small domains filled with the composite material. However, if the angle of rotation of the cells of the composite is not introduced as a design variable, considerable areas of the resulting structure are made of a composite of intermediate density. The resulting designs may still be of interest if composite structures are to be produced, but for definition of shape the introduction of the cell rotation is crucial. This property is illustrated in Fig. 13.

The optimization method results in material distributions that give a good definition of shape, but even with the cell rotation taken into account the optimal design still includes some small areas of composite material. If such composites with microscopic voids are difficult or impossible to produce, it will be convenient to introduce some method for getting designs with no composites from the optimal designs computed using composites. This can be done interactively, where the designer chooses the final shape of the element to be produced from the shape produced by the optimal design procedure just described. An alternative is to numerically perform a lumping process where in each element of the discretized structure it is decided whether this element should be solid or void in the macroscopic scale. The lumping is based on the distribution of material computed in the optimal design procedure, with a cut-off value for material/void defined from the requirement that the volume should be unchanged. Such a lumping method has also been employed by Cheng and Olhoff [18], and, as in their work, the lumping in the present case has in most cases insignificant influence on the performance of the structure. This means that the lumping process is an efficient method for computing nearly optimal, practical designs. Some examples are given in Fig. 14. In some cases, however, this crude lumping process can lead to a break-up of the structure and isolated pieces of material, in which case a final acceptable design must be done manually.

Finally, the shape optimization method proposed here was compared with a standard boundary variations technique, as described in [8]. For a considerably higher computing cost this method gives comparable values of the criteria as well as very similar shapes for higher volume fractions, where it is unnecessary to include holes in the structure, while for little 

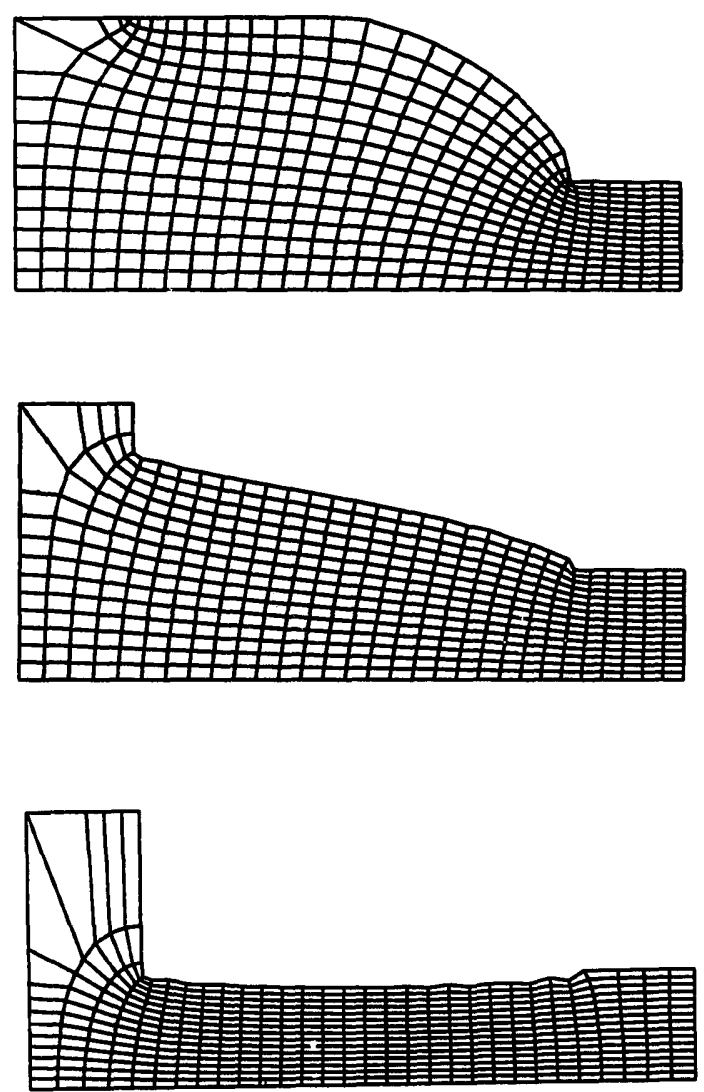

Fig. 15. Results obtained by boundary variations. Case A, with volumes $91 \%, 64 \%$ and $36 \%$.

available material the resulting shapes are different and the compliance for the method proposed here is significantly smaller. In the ideal situation, the two methods should be combined, by using the density method described here as a preprocessor for a boundary movement technique in order to obtain the smooth and detailed optimal shape of a structure (see Fig. 15).

\section{Conclusions}

As shown above the proposed optimization method can provide the optimal shape as well as the topology of a mechanical element. The method is a material distribution method, based on the use of an artificial composite material with microscopic voids.

Further investigation of the sensitivity of the results with regard to choice of particular microstructure should be carried out and extension of the present method to three-dimensional problems is of great importance for the applicability to real-life problems. It is noted that the boundary variations method for three-dimensional structures is very difficult to implement because of the difficulties encountered in representing the boundary surfaces. In other words, extending the boundary variations method to three-dimensional problems is not straightforward but the present method should easily be extended. 


\section{Acknowledgment}

This work has been supported by The Technical University of Denmark through a guest professorship (N.K.). Support from the Danish Technical Research Council, grants nos. 16-4058.M (M.P.B. and N.K.) and 16-4246.M (M.P.B.), and support from NASA Lewis Research Center NAG3-661 (N.K.), is also gratefully acknowledged.

\section{References}

[1] R.T. Haftka and R.V. Gandhi, Structural shape optimization-A survey, Comput. Meths. Appl. Mech. Engrg. 57 (1986) 91-106.

[2] O. Pironneau, Optimal Shape Design for Elliptic Systems (Springer, Berlin, 1984).

[3] E. Schanck, An optimization procedure for stress concentration by the finite element technique, Internat. J. Numer. Meths. Engrg. 14 (1979) 115-124.

[4] N. Kikuchi, K.Y. Chung, T. Torigaki and J.E. Taylor, Adaptive finite element methods for shape optimization of linearly elastic structures, Comput. Meths. Appl. Mech. Engrg. 57 (1986) 67-91.

[5] V. Tvergaard, On the optimum shape of a fillet in a flat bar with restrictions, in: A. Sawczuk and Z. Mroz, eds., Optimization in Structural Design (Springer, New York, 1975) 181-195; also in: Proceedings IUTAM Symposium on Optimization in Structural Design (Springer, Berlin, 1973).

[6] M.E. Botkin and J.A. Bennett, Shape optimization of three-dimensional folded-plate structures, AIAA J. 23-11 (1985) 1804-1810.

[7] V. Braibant and C. Fleury, Shape optimal design using B-splines, Comput. Meths. Appl. Mech. Engrg. 44 (1984) 247-267.

[8] H. Rodrigues, Shape optimal design of elastic bodies using a mixed variational formulation, Comput. Meths. Appl. Mech. Engrg. 69 (1988) 29-44.

[9] E.J. Haug, K.K. Choi and V. Komkov, Design Sensitivity Analysis of Structural Systems (Academic Press, New York, 1986).

[10] K.K. Choi and E.J. Haug, Shape design sensitivity analysis of elastic structures, J. Structural Mech. 11(2) (1983) 231-269.

[11] K.K. Choi and K.K. Seong, A domain method for shape design sensitivity analysis of built-up structures, Comput. Meths. Appl. Mech. Engrg. 57 (1986) 1-16.

[12] B. Rousselet and E.J. Haug, Design sensitivity analysis in structural mechanics, III. Effects of shape variation, J. Structural Mech. 10(3) (1983) 273-310.

[13] J.P. Zolesio, The material derivative (or speed) method for shape optimization, in: E.J. Haug and J. Cea, eds., Optimization of Distributed Parameter Structures (Sijthoff-Noordhoff, Alphen aan den Rijı, The Netherlands, 1981) 1152-1194.

[14] J. Simon, Differentiation with respect to the domain in boundary value problems, Numer. Funct. Anal. Optim. 2 (1980) 649-687.

[15] R.B. Haber, A new variational approach to structural shape design sensitivity analysis, in: C.A. Mota Soares, ed., Computer Aided Optimal Design: Structural and Mechanical Systems (Springer, Berlin, 1987) 573-588.

[16] D. Chenais, On the existence of a solution in a domain identification problem, J. Math. Anal. Appl. 52(2) (1975) 189-219.

[17] K.T. Cheng and N. Olhoff, An Investigation Concerning Optimal Design of Solid Elastic Plates, Internat. J. Solids and Structures 16(3) (1981) 305-323.

[18] K.T. Cheng and N. Olhoff, Regularized Formulation for Optimal Design of Axisymmetric Plates, Internat. J. Solids and Structures 18(2) (1982) 153-170.

[19] J. Cea, A. Gioan and J. Michel, Quelques Resultat sur l'identification de domaines, Calcolo III-IV (1973).

[20] L. Tartar, Estimation de coefficients homogeneises, Lecture Notes in Mathematics 704 (Springer, Berlin, 1977) 364-373. 
[21] M.P. Bendsøe, Generalized plate models and optimal design, in J.L. Eriksen, D. Kinderlehrer, R. Kohn and J.-L. Lions, eds., Homogenization and effect moduli of materials and media, The IMA Volumes in Mathematics and Its Applications (Springer, Berlin, 1986) 1-26.

[22] L.V. Gibiansky and A.V. Cherkaev, Design of composite plates of extremal rigidity, Rept. No. 914, A.F. Ioffe Physical Technical Institute, Academy of Sciences of the USSR, Leningrad, 1984 (in Russian).

[23] K.A. Lurie, A.V. Fedorov and A.V. Cherkaev, Regularization of optimal design problems for bars and plates, Parts I and II, J. Optim. Theory Appl. 37(4) (1982) 499-521, 523-543.

[24] G.I.N. Rozvany, T.G. Ong, W.T. Szeto, N. Olhoff and M.P. Bendsøe, Least-weight design of perforated plates, Internat. J. Solids and Structures 23 (1987) 521-536 (Part I), 537-550 (Part II).

[25] J. Goodman, R.V. Kohn and L. Reyna, Numerical study of a relaxed variational problem from optimal design, Comput. Meths. Appl. Mech. Engrg. 57 (1986) 107-127.

[26] R.V. Kohn and G. Strang, Optimal design in elasticity and plasticity, Internat. J. Numer. Meths. Engrg. 22 (1986) 183-188.

[27] A. Zochowski, The design of a two-dimensional domain, Mech. Struct. Mach. 16 (1988) (to appear).

[28] A. Bensousson, J.-L. Lions and G. Papanicolaou, Asymptotic Analysis for Periodic Structures (NorthHolland, Amsterdam, 1978).

[29] G. Duvaut, Analyse fonctionnelle et mecanique des milieus continus. Application a l'etude des materiaux composites elastiques a structure periodique-homogeneisation, in: Proceedings 14th IUTAM Congress, Delft, The Netherlands, 1976 (North-Holland, Amsterdam, 1976) 119-132.

[30] E. Sanchez-Palencia, Non-Homogeneous Media and Vibration Theory, Lecture Notes in Physics 127 (3pringer, Berlin, 1980).

[31] F. Murat and L. Tartar, Calcul des variations et homogeneisation, in: Les Methodes de l'Homogeneisation: Theorie et Applications en Physique, Coll. de la Dir. des Etudes et Recherches de Electricite de France, Eyrolles, Paris (1985) 319-370.

[32] M. Avellaneda: Optimal bounds and microgeometries for elastic two-phase composites, SIAM J. Appl. Math. 47 (1987) 1216-1228.

[33] G.A. Francfort and F. Murat, Homogenization and optimal bounds in linear elasticity. Arch. Rat. Mech. Anal. 94 (1986) 307-334.

[34] R.V. Kohn and G.W. Milton, On bounding the effective conductivity of anisotropic composites, in: J.L. Eriksen, D. Kinderlehrer, R. Kohn and J.-L. Lions, eds., Homogenization and Effective Moduli of Materials and Media. The IMA Volumes in Mathematics and Its Applications 1 (Springer, Berlin, 1986) 97-126.

[35] K.A. Lurie and A.V. Cherkaev, Exact estimates of conductivity of composites formed by two isotropically conducting media taken in prescribed proportion, Proc. Roy. Soc. Edinburgh 99A (1984) 71-78.

[36] R.V. Kohn and M. Vogelius, A new model for thin plates with rapidly varying thickness, Internat. J. Solids and Structures 29(4) (1984) 333-350.

[37] R.V. Kohn and M. Vogelius, A new model for thin plates with rapidly varying thickness II: A convergence proof, Quart. Appl. Math. 43 (1985) 1-22.

[38] R.V. Kohn and M. Vogelius, A new model for thin plates with rapidly varying thickness III: Comparison of different scalings, Quart. Appl. Math. 44 (1986) 35-48.

[39] R.V. Kohn and G. Strang, Optimal design and relaxation of variational problems, Comm. Pure Appl. Math. 39 (1986) 1-25 (Part I), 139-182 (Part II), and 353-377 (Part III).

[40] E. Bonnetier and $M$. Vogelius, Relaxation of a compliance functional for a plate optimization problem, Preprint, University of Maryland, College Park, MD, 1986.

[41] M.P. Bendsøe, On obtaining a solution to optimization problems for solid, elastic plates by restriction of the design space, J. Structural Mech. 11 (1983) 501-521.

[42] J. Francu, Homogenization of linear elasticity equations, Apl. Mat. 27 (1982) 96-117.

[43] G.A. Josifjan, O.A. Oleinik and G.P. Panasenko, Asymptotic expansion of a solution of the system of elasticity theory with periodic rapidly oscillating coefficients, Soviet Math. Dokl. 26 (1982) 290-294.

[44] J.F. Bourgat, Numerical Experiments of the Homogenization Method for Operators with Periodic Coefficients, Lecture Note in Mathematics 704 (Springer, Berlin, 1977) 330-356.

[45] J.M. Guedes, Homogenization in linear elasticity theory, Preprint, University of Michigan, Ann Arbor, MI, 1987. 
[46] D. Cioranescu and J. Saint Jean Paulin, Homogenization in open sets with holes, J. Math. Anal. Appl. 71 (1979) 590-607.

[47] N. Olhoff and J.E. Taylor, On structural optimization, J. Appl. Mech. 50 (1983) 1134-1151.

[48] E.S. Kristensen and N.F. Madsen, On the optimum shape of fillets in plates subjected to multiple in-plane loading cases, Internat. J. Numer. Meths. Engrg. 10 (1976) 1007-1009.

[49] P. Pedersen and C.L. Laursen, Design for minimum stress concentration by finite elements and linear programming, J. Structural Mech. 10(4) (1983) 375-391.

[50] C.A.M. Soares, H.C. Rodrigues, L.M.O. Faria and E.J. Haug, Optimization of the geometry of shafts using boundary elements, ASME J. Mechanics, Transmissions and Automation in Design 106(2) 199-203.

[51] M.P. Rossow and J.E. Taylor, A finite element method for the optimal design of variable thickness sheets, AIAA J. 11 (1973) 1566-1569.

[52] G.I.N. Rozvany, Structural layout theory - the present state of knowledge, in: E. Atrek, R.H. Gallagher, K.M. Ragsdell and O.C. Zienkiewicz, eds., Directions in Optimum Structural Design (Wiley, Chichester, U.K., 1984) Ch. 7. 